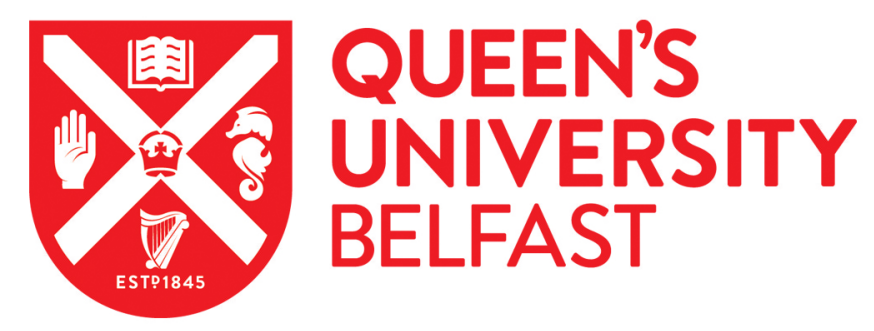

\title{
Rapid ex vivo molecular fingerprinting of biofluids using laser-assisted rapid evaporative ionization mass spectrometry
}

Plekhova, V., Van Meulebroek, L., De Graeve, M., Perdones-Montero, A., De Spiegeleer, M., De Paepe, E., Van de Walle, E., Takats, Z., Cameron, S. J. S., \& Vanhaecke, L. (2021). Rapid ex vivo molecular fingerprinting of biofluids using laser-assisted rapid evaporative ionization mass spectrometry. Nature Protocols. https://doi.org/10.1038/s41596-021-00580-8

Published in:

Nature Protocols

Document Version:

Peer reviewed version

Queen's University Belfast - Research Portal:

Link to publication record in Queen's University Belfast Research Portal

Publisher rights

Copyright 2021, the Authors.

This work is made available online in accordance with the publisher's policies. Please refer to any applicable terms of use of the publisher.

\section{General rights}

Copyright for the publications made accessible via the Queen's University Belfast Research Portal is retained by the author(s) and / or other copyright owners and it is a condition of accessing these publications that users recognise and abide by the legal requirements associated with these rights.

Take down policy

The Research Portal is Queen's institutional repository that provides access to Queen's research output. Every effort has been made to ensure that content in the Research Portal does not infringe any person's rights, or applicable UK laws. If you discover content in the Research Portal that you believe breaches copyright or violates any law, please contact openaccess@qub.ac.uk. 
Rapid ex vivo molecular fingerprinting of biofluids using laser assisted rapid evaporative ionization mass spectrometry

85

Vera Plekhova ${ }^{1 *}$, Lieven Van Meulebroek ${ }^{1,2^{*}}$, Marilyn De Graeve ${ }^{1}$, Alvaro PerdonesMontero $^{3}$, Margot De Spiegeleer ${ }^{1}$, Ellen De Paepe ${ }^{1}$, Emma Vande Walle $^{1}$, Zoltan Takats ${ }^{3}$,

89

* Equal first author contributions

$91 \quad$ \# Equal last author contributions

92

$93{ }^{1}$ Laboratory of Chemical Analysis, Ghent University, 9820 Merelbeke, Belgium.

$94{ }^{2}$ ProDigest BV, Technologiepark-Zwijnaarde 82, 9052 Zwijnaarde, Belgium.

$95{ }^{3}$ Department of Surgery and Cancer, Imperial College London, London SW7 2AZ, U.K.

$96{ }^{4}$ School of Biological Sciences, Institute for Global Food Security, Queen's University Belfast, 97 BT9 5DL Belfast, Northern Ireland, U.K.

98

Of the many metabolites that are are involved in any clinical condition, only a narrow range of biomarkers is currently being used in the clinical setting. A key to personalised medicine would be to extend this range. Metabolic fingerprinting provides a more comprehensive insight, but many methods used for metabolomics analysis are too complex and time-consuming to be diagnostically useful. Here, a rapid evaporative ionization mass spectrometry (REIMS) system for direct ex vivo real-time analysis of

105 biofluids with minor sample pre-treatment is detailed. The REIMS can be linked to various laser wavelength systems (such as OPO or $\mathrm{CO}_{2}$ laser) and with automation for high- 
107 throughput analysis. Laser-induced sample evaporation occurs within seconds through

108 radiative heating with the plume guided to the MS instrument. The presented procedure

109 includes (i) laser set-up with automation, (ii) analysis of biofluids

110 (blood/urine/stool/saliva/sputum/breast milk), and (iii) data analysis. We provide the

111 optimal settings for biofluid analysis and quality control, enabling sensitive, precise and

112 robust analysis. Using the automated set-up, 96 samples can be analysed in ca. 35-40

113 minutes per ionization mode, with no intervention required. Metabolic fingerprints

114 enclose about $\mathbf{2 0 0 0}$ to $\mathbf{4 0 0 0}$ features, for which relative quantification can be achieved at

115 high repeatability when total ion current normalization is applied. With saliva and feces as

116 example matrices, more than $\mathbf{7 0 \%}$ of features had a coefficient of variance $\leq \mathbf{3 0} \%$.

117 However, to achieve acceptable long-term reproducibility, additional normalizations by

118 e.g. LOESS are recommended, especially for positive ionization.

Introduction

121 To date, metabolic fingerprinting of human biological fluids is most typically accomplished

122 through high-resolution mass spectrometry (HRMS) in hyphenation with a chromatographic

123 separation technique such as capillary electrophoresis, liquid or gas chromatography ${ }^{1,2}$. This

124 analytical approach allows for the sensitive and selective measurement of low-molecular

125 metabolites (< $1500 \mathrm{Da}$ ), generating a metabolic read-out that can be used to gain

126 interesting insights in biological and (patho)physiological processes ${ }^{3,4}$. More specifically,

127 biomarker metabolites and biological pathways can be shown to be associated with

128 particular phenotypes of human health and disease ${ }^{5}$. This approach does, however, have

129 some substantial shortcomings that strongly restrict the general application potential; i.e.

130 protocols are often laborious and involving elaborate sample preparations, which contribute 
131 to relatively low sample throughput rates and high costs as well as a significant risk for

132 artefacts such as metabolite degradation, causing variable or poor recovery ${ }^{2}$. These

133 requirements and limitations mean that metabolic fingerprinting by hyphenated HRMS is

134 not practical for e.g. point-of-care testing in clinical practice. Indeed, in that setting, there is

135 a high need for simplicity and automation to analyze large sample batches, there is only

136 limited time for sample analysis, the measurements are of an individualized nature, and the

137 physical sample size available is often limited ${ }^{6,7}$.

138 It is therefore productive to explore alternative analytical strategies such as ambient 139 ionization mass spectrometry that are able to produce metabolic fingerprints, but that don't

140 require extensive sample preparation or chromatographic separation. With ambient

141 ionization techniques, samples are analysed in their native environment or after minimal

142 pre-treatment; and gas-phase ions are generated under open-air conditions and directly

143 introduced into the mass spectrometer ${ }^{6,8}$. Traditionally, ambient ionization has been

144 achieved through various desorption ionization methods such as ambient pressure matrix-

145 assisted laser desorption (AP-MALDI), direct analysis in real-time mass spectrometry (DART-

$146 \mathrm{MS})$, desorption electrospray ionization (DESI), and secondary ionization mass spectrometry

147 (SIMS). These approaches have been used successfully in the analysis of intact (ex vivo)

148 biological tissues, but are not suitable for ionising living tissues. There, thermal evaporation

149 techniques have become the norm, including thermal electrosurgery and laser surgery ${ }^{9,10}$.

150 These have mostly been used to determine the position of the borders between malignant

151 tumours and healthy tissue ${ }^{11}$.

152 Only recently, laser assisted ambient ionization mass spectrometry (LA-REIMS, laser assisted

153 rapid evaporative ionization MS) has also been exploited (by the authors of this paper) for

154 rapid, real-time fingerprinting of various human biofluids, i.e. saliva, urine, feces, sputum, 
155 breast milk and plasma ${ }^{7,12,13}$. In this approach, the process of laser ablation is based on the 156 laser-emitted infrared wavelength regime that excites the most intense vibrational band

157 (oxygen-hydrogen stretching mode) of the water molecules that are highly present in the 158 respective biological fluids, initiating matrix-assisted desorption and ionization of intact

159 biomolecules ${ }^{14}$. It should be noted that, in comparison with other techniques, laser assisted

160 ionization does not require the usage of solvents (versus e.g. DESI), high voltages or gas

161 flows (e.g. DART, 2 to $4 \mathrm{kV}$ discharge needle voltage and 1.5 to $3.0 \mathrm{~L} / \mathrm{min}$ gas flow rate), nor

162 does it require direct contact with the sample (e.g. hand-held sampling devices such as the

163 iKnife that are typically used to cut through tissue and rapidly heat it, to generate a vapor) ${ }^{7}$.

164 The approach that is used in this protocol is very similar to the iKnife, but the fact that there

165 is no probe that has direct contact with the samples means that the cleaning requirements

166 and the risk of sample-to-sample contamination are substantially lower. These are

167 considered essential conditions for platform automation.

168 In LA-REIMS, no pre-treatment is needed for the analysis of fresh feces, urine, plasma,

169 breast milk and sputum, and only centrifugation and filtration are required for saliva 170 analysis. As such, fingerprinting of biofluids by LA-REIMS could generally be achieved within

171 less than $1 \mathrm{~min}$ per sample ${ }^{7,12,13}$. In addition, we assume that artefactual metabolite 172 modifications are less likely to occur in experiments with fewer sample processing steps and 173 shorter analytical times. In comparison with hyphenated techniques, LA-REIMS has 174 substantially fewer analytical biases relating to sample pre-treatment, extraction and 175 storage (i.e. samples are analysed in or very close to their native condition), or resulting from 176 chromatographic and mass spectrometric drifts (i.e. the analysis times are much shorter).

177 Without chromatographic separation, however, metabolome coverage is likely to be lower 178 as characterization of metabolite-associated features is based on mass measurements only. 
179 As such, it will for example not be possible to separate isomers and isobars. This also limits 180 the possibilities for quantification. Data from LA-REIMS are thus not as comprehensive as

181 those from conventional metabolic fingerprinting approaches (i.e., UHPLC-HRMS) ${ }^{4}$. In 182 addition, secondary ionization of ablated metabolites at the rapid evaporative ionization 183 (REI) collision surface may also suffer from ion suppression, which has adverse effects on 184 sentivity and quantification accuracy ${ }^{15}$. As such, biofluid metabolomics by LA-REIMS may 185 primarily find acceptance in first-line segregation of samples based on distinctive fingerprints 186 and according to (patho)physiological state, as has been demonstrated by a number of 187 clinical studies ${ }^{7,12,13}$. For a more detailed read-out of the metabolome, conventional 188 hyphenated techniques can be used to achieve advanced separation and in-depth 189 characterization as well as quantification of metabolites.

190 Laser ablation ambient ionization mass spectrometry has also been described for rapid 191 metabolic fingerprinting of tissue (e.g. by Fatou et al., 2016) ${ }^{14}$ and is the subject of another 192 Nature Protocol (i.e., Ogrinc et al., 2019) ${ }^{8}$. This manuscript, therefore, focuses on rapid 193 metabolic fingerprinting of biofluids and captures the experience gained in multiple studies 194 published by the involved authors. Key procedural differences are found at many stages of 195 the protocol: at the level of the instrumental set-up (e.g. the need for a T-shaped connector 196 piece at the REI-source to concentrate the low metabolite concentrations as present in the 197 aerosol), instrumental settings (both with respect to the laser as well as the REI-mass 198 spectrometer) as well as biofluid sample handlings (e.g. specific volumes, pre-treatments, 199 and cleaning procedures). This work also describes how to adapt the method for 200 automation.

201 
The LA-REIMS methodology (Fig. 1) comprises direct ablation following laser irradiation and

204 subsequent aerosolization from the sample surface under analysis. In this protocol, an

$205 \mathrm{Nd}$ :YAG laser (operating wavelength of $2.94 \mu \mathrm{m}$ ) and $\mathrm{CO}_{2}$ laser (operating wavelength of

$20610.64 \mu \mathrm{m})$ for laser irradiation are described. A detailed comparison between these laser

207 types can be found in the study of Schäfer et al. $(2011)^{16}$ that reported on the reproducibility

208 of analysis, induction of photochemical processes, coagulative properties, and uniformity of

209 the generated mass spectra according to wavelength, specifically assessed for tissue. For

210 biofluid analysis, own research indicated the suitability of both laser types to achieve

211 comprehensive metabolic fingerprinting, however, without having made explicit

212 comparisons. The energy of the emitted infrared laser beam is absorbed by the water

213 molecules, abundantly present in the biological matrices, as a result of the stretching

214 vibrations of the hydroxyl groups ${ }^{17}$. This desorption and ionization mechanism has the

215 unique advantage of being able to exploit the presence of abundant endogenous water ${ }^{18,19}$.

216 The setup also features an MS-source (i.e., REI-source) that is specifically designed to

217 efficiently draw the produced aerosol through an aerosol vent transmission tube

218 (millisecond timescale) into the deep vacuum region of the MS-instrumentation. This tube

219 interfaces the MS-source by means of an orthogonally mounted T-shaped connector piece

220 and designated insert that is surrounding the aerosol inlet tube. In the source, declustering

221 of the aerosol, comprising desorbed droplets of both positive and negative analyte ions,

222 takes place by collision onto a heated impactor, whereby individual molecular species are

223 generated. Therefore, LA-REIMS is a two-step technique involving desorptive release of the

224 chemical components and secondary ionization mechanisms ${ }^{18}$. Ionization is assisted by a

225 solvent matrix, for example isopropylalcohol, that is provided at the REI-source ${ }^{20}$. This

226 solvent plays a MALDI-like role in that it reduces intermolecular forces and improves ion 
227 formation. More specifically, the polar protic nature of isopropylalcohol is useful in 228 dissolving lipid species and as such, enables the formation of individual molecular ions via a

229 collision driven ion release mechanism (heated impactor) which ultimately enhances the 230 signal-to-noise ratio ${ }^{21}$. Moreover, aerosol dilution reduces fouling at the inlet capillary 231 resulting in better spectral classifications and allowing the instrument to run under high232 throughput conditions. The flow rate of the solvent matrix is optimized for every biological 233 matrix type (e.g. $150 \mu \mathrm{L} / \mathrm{min}$ for feces) and can contain a 'lock mass' external calibration 234 compound (e.g. $10 \mathrm{ng} / \mathrm{mL}$ leucine-enkephalin).

235 As a measure of securing consistency in the analysis process through reduction of operator236 related factors and to further increase the analysis speed, an automated versatile multiwell 237 plate-based platform was incorporated in the standard REIMS workflow. The latter serves to 238 introduce fully remote and automated fingerprinting of a large number (up to 576) of small 239 volumes of biological material in a fraction of time. Additionally, as positioning the samples 240 inside the restricted area of each well results in a more uniform sample distribution 241 compared to the open area of the microscope slide, the microplate configuration 242 contributes further benefits to standardization of analyses. A flowchart (Fig. 2) presenting 243 the entire procedure from sample collection to data processing is provided.

\section{Applications of the method}

246 Whereas blood is the most sampled biofluid in clinical research, diverse alternatives are 247 available that can be accessed non-invasively and offer an at least equally rich and 248 chemically diverse metabolome coverage, reflective of various biological processes. Among 249 those are urine (a key harbor of metabolic waste products ${ }^{11}$ addressed in e.g. illicit drug screening), saliva (parallels metabolic alterations occurring in blood and applied in studying 
251 e.g. obesity ${ }^{22}$ ), stool (ideally suited to study the diet-microbiome-metabolome axis in gastro-

252 intestinal disorders, e.g. type 2 diabetes ${ }^{23}$ ), and sputum (a means to study 253 bacterial activity within the airways, e.g. in cystic fibrosis ${ }^{24}$ ). LA-REIMS provides an almost 254 instantaneous fingerprinting strategy without (or hardly) damaging the analysed biofluids 255 and as such allows for cross-evaluation with conventional methodologies. In this regard, the 256 LA-REIMS platform described presently, has recently demonstrated rapid fingerprinting of 257 saliva and feces, each complemented by metabolic profiling and cross-evaluation against 258 established UHPLC-HRMS metabolomics and lipidomics platforms ${ }^{4,25,26}$ for in-depth 259 biological interpretation. The ambient ex vivo analysis of both cited biofluids allowed the 260 establishment of validated multivariate models that were predictive for assigning metabolic 261 perturbations according to pathophysiology, i.e. weight and glycaemic state, and provided 262 general classification accuracies of $97.1 \%$ and $90.5 \%$, respectively ${ }^{4}$. Thus, LA-REIMS 263 represents an expedient strategy in first-line distinctive metabotyping based on easy to 264 obtain biofluids ${ }^{12}$.

\section{Limitations and potential improvements of the protocol}

267 The LA-REIMS method presented here suffers similar limitations as many other methods in 268 the field of ambient ionization mass spectrometry and wider fields of direct and flow 269 infusion $\mathrm{MS}^{27}$. These limitations arise from the removal of chromatographic separation of 270 the chemical contents of a sample, prior to ionization. For LA-REIMS, this means that ion

271 formation for a sample's metabolic fingerprint is condensed into a two to eight second time

272 frame compared to hyphenated methods, such as LC- and GC-MS, where molecules are 273 introduced for ion generation over a typical 10 to 20 minute period ${ }^{7}$. Due to ion suppression 274 effects, this will likely result in a lower sensitivity of the LA-REIMS method compared to 
275 hyphenated methods, even when the same high resolution mass spectrometer is used for 276 analysis. The usage of internal or external standards may provide a relevant strategy to 277 address this issue, although it is not possible to reach sufficient representativeness for all 278 metabolome constituents. In addition, the lack of chromatographic separation also hampers 279 the structural identification of features. Indeed, without having retention time as an 280 orthogonal separator, it is not straightforward to cluster different $\mathrm{m} / \mathrm{z}$-features that are 281 originating from the same metabolite (e.g. $\left[\mathrm{M}+\mathrm{H}^{+}\right]$and $\left[\mathrm{M}-\mathrm{H}_{2} \mathrm{O}+\mathrm{H}^{+}\right]$ionization adducts) nor is 282 it possible to distinguish metabolites that are represented by identical or similar $\mathrm{m} / \mathrm{z}$-values 283 (i.e., isobars or structural and stereoisomers) ${ }^{28}$. This can be somewhat overcome through 284 usage of the instrument's tandem MS functionality and the consultation of repositories with 285 fragmentation data. However, interpretation of fragmentation data is not evident as 286 metabolites with identical or similar $m / z$-values will be subjected to the the same selection 287 and fragmentation event, resulting in intertwined fragmentation $\mathrm{m} / \mathrm{z}$-spectra. Moreover, the 288 inclusion of fragmentation scan events during the short period of the burn is at the expense 289 of the full-scan events, which may have adverse consequence for the sensitivity and 290 quantification accuracy. Hence, the information that is obtained from chromatographic 291 separation and that is supportive for identification cannot be easily replaced. One strategy 292 to improve the analytical resolution of LA-REIMS, being equivalent to chromatographic 293 separation, could be the application of ion mobility separation ${ }^{29}$. As separation occurs on the 294 millisecond scale, it complements the method's rapid analysis time and provides a second 295 dimension to analytical resolution. Moreover, it is does not interfer with the full-scan events. 296 Usage of ion mobility would, however, add extra complexity to instrumental operation and 297 maintenance, which not fully aligns with the purpose of LA-REIMS to translate metabolomics 298 into clinical practice and for point-of-care implementations. In this respect, as earlier noted, 
LA-REIMS is merely proposed as a strategy for first-line seggragation of samples, without in-

300

301

302 depth biological-mechanistic explorations.

Further benefits to the LA-REIMS method could be achieved through alternative means of sample heating and evaporation, particularly with regard to the pulsatile and wavelength characteristics of lasers. It may be possible to target specific chemical classes through optimisation of laser choice, reducing ionization competition and improving analytical sensitivity. Similar benefits could be pursued through modification of the solvent matrix including different polarities, or through the addition of additives to enhance the ionization of particular analytical targets ${ }^{30}$.

\section{Experimental design}

\section{Sample preparation}

The method allows for the analysis of freshly collected as well as thawed specimens (Box 1). If samples cannot be analysed immediately upon collection, storage at $-80{ }^{\circ} \mathrm{C}$ is recommended to preserve the metabolic fingerprint. Aside from temperature conditions, maximal storage duration should be considered when designing the experiment as well ${ }^{31,32}$. Liquid samples can be aliquoted and frozen without treatment, while semi-solid matrices (such as feces) should preferentially undergo lyophilisation ${ }^{33}$ prior to storage.

\section{Quality control samples}

In addition to samples under investigation, external quality control (eQC) samples should be prepared for instrument conditioning and internal quality control (iQC) samples for monitoring instrumental performance and possible data normalization. For cohort studies, these QC sample can be prepared by aliquoting and thoroughly mixing equal volumes from a selection of representative samples (i.e., including the assessed disease states, age 
categories, treatments, etc.) or, preferably, from all biological samples. The final volume of

324 the QC blend should allow to include ten eQCs per ionization mode at the beginning of the

sequence and two iQCs every 20 to 40 analytical runs (depending on batch size and available sample volume $)^{12}$. On the other hand, a reference material that is not directly derived from

the sample batch for analysis can be used as well, being self-assembled from any excess experimental samples or commercially available (e.g. pooled human serum, MP Biomedicals, cat.no 2930149). This may be particularly useful for discontinuous LA-REIMS analysis of samples across several analytical batches, e.g. for general metabotyping or specific diagnosis 331 of patients.

BOX 1 Sample preparation • Timing 0-15 min

This box describes sample handling and preparation for analysis of freshly collected as well as previously frozen samples.

! CAUTION When using human samples in experimental settings, all necessary local and global ethical regulations should be fulfilled.

\section{Additional materials}

- Plastic microcentrifuge tubes (2 mL, Eppendorf, Germany, cat. no. 0030120094)

- Microcentrifuge (MicroCL 17, Thermo Fisher Scientific, USA, cat. no. 75002406)

- Analytical balance (ML104T/00, Mettler Toledo, USA, cat. no. 30243394) 
- $1 \mathrm{~mL}$ Luer slip syringes (Becton Dickinson, USA)

- Polyamide membrane filters (CHROMAFIL ${ }^{\circledR}, 13 \mathrm{~mm}$ diameter, $0.45 \mu \mathrm{m}$ pore size; Macherey-Nagel, Germany, cat. no. 91652)

- Sartorius Arium 661 UV water purification system (Sartorius, Germany, cat. no. $\mathrm{H}_{2}$ OPRO-UV-B-TOC)

- Vortex shaker IKA vortex genius 3 (IKA-Werke GmbH \& Co. KG, Germany, cat. no. 0003340000)

\section{Procedure}

$\triangle$ CRITICAL STEP All frozen samples should be defrosted at $4{ }^{\circ} \mathrm{C}$ shortly before described pre-treatment procedures.

\section{Saliva}

1. Vortex raw samples (freshly collected or defrosted) during $30 \mathrm{~s}$ at $400 \times \mathrm{g}$ and room temperature $\left(25^{\circ} \mathrm{C}\right)$ to ensure homogenization.

2. Transfer $450 \mu \mathrm{L}$ of each sample into separate microcentrifuge tubes.

3. Centrifuge at $17000 \times \mathrm{g}$ for $5 \mathrm{~min}$ at room temperature.

4. Collect all resulting supernatant in $1 \mathrm{~mL}$ syringe and pass through polyamide membrane filter into new microcentrifuge tube. This can be analysed immediately or stored at $-80{ }^{\circ} \mathrm{C}$, preferably for less than 1 month ${ }^{34}$.

\section{Feces}

1. Freshly collected stool samples can be homogenized manually with a sterile spatula or 
using commercially available homogenizers (e.g. Stomacher $\left.{ }^{\circledR}\right)$ according to manufacturer guidelines. Following homogenization, these samples are ready for LA-REIMS analysis.

2. If prolonged storage of fecal samples is required, consider lyophilising the samples. They can then be stored at $-80{ }^{\circ} \mathrm{C}$, preferably for less than 2 months ${ }^{31}$.

3. When preparing the lyophilised samples for LA-REIMS analysis:

(i) Weigh $200( \pm 1) \mathrm{mg}$ of lyophilized homogenized feces in $1.5 \mathrm{~mL}$ microcentrifuge tube.

(ii) Add $600 \mu \mathrm{L}$ of ultrapure water.

(iii) Thoroughly mix by vortexing for $60 \mathrm{~s}$ at $400 \mathrm{xg}$ (if homogeneity is not achieved, additionally a sterile spatula may be used) and keep at $4{ }^{\circ} \mathrm{C}$ prior to analysis.

\section{Urine and blood plasma}

1. Freshly collected samples do not require any pre-treatment. If analysis is not performed immediately, samples can be stored at $-80^{\circ} \mathrm{C}$ for preferably less than 1 month ${ }^{35,36}$.

2. Defrosted samples require a homogenization step, by vortexing at $400 \times \mathrm{g}$ for $30 \mathrm{~s}$ and keep them at $4{ }^{\circ} \mathrm{C}$ prior to analysis.

\section{Human milk}

1. Homogenise freshly collected samples by vortexing at $400 \times \mathrm{g}$ for $30 \mathrm{~s}$. These can be analysed directly or frozen for storage at $-80^{\circ} \mathrm{C}$ for preferably less than 3 months ${ }^{37}$. Vortex after defrosting at $400 \times \mathrm{g}$ for $30 \mathrm{~s}$ and keep at $4{ }^{\circ} \mathrm{C}$ prior to analysis.

2. If the analytical focus is fat and complex lipids, perform refrigerated centrifugation $\left(4^{\circ} \mathrm{C}\right)$ for $5 \mathrm{~min}$ at $3000 \times \mathrm{g}$ to create a fat layer that can be directly analysed by LA-REIMS. If required, the fatty layer can be removed from the aqueous phase with a sterile single-use $10 \mu \mathrm{L}$ microbiological inoculating loop and transferred to a sterile microcentrifuge tube for separate analysis. 


\section{Sputum}

1. Freshly collected samples do not require any pre-treatment. If analysis is not performed immediately, frozen samples can be stored at $-80{ }^{\circ} \mathrm{C}$ for at least 1 month ${ }^{38}$. Defrost at $4{ }^{\circ} \mathrm{C}$ prior to LA-REIMS analysis.

C. If desired for easier homogenization of the sample matrix, sputum can be treated with Sputasol (\#SR0233, Oxoid, ThermoFisher Scientific, USA) following standard manufacturer's instructions. This can be completed on freshly collected samples prior to analysis or prior to storage at $-80^{\circ} \mathrm{C}$. Homogenisation may be necessary where visible colour heterogeneity exists within the sample or variations in consistency across the sample is noted, and which is not resolved through vortex mixing. During experimental design, consideration should be given to whether sample homogenization with Sputasol should be undertaken regardless of sample appearance as it would be a likely source of bias to only treat a selection of samples.

\section{Quality control samples}

1. Define how many analytical runs will be considered for the LA-REIMS experiment and calculate how many $Q C$ runs are needed. Guidelines regarding the number and frequency of eQC and iQC samples have been defined previously (section 'Quality control samples').

2. For determination of the total volume needed, take into account the recommended starting-volumes per matrix type (see section 'Procedure' below) and consider a separate glass microscope slide / well for every QC run.

3. Establish a QC-pool by taking equal aliquots from (a selection of) representative samples or by considering general reference material. Vortex the QC-pool 
thoroughly.

4. Store the QC-pool at $-80{ }^{\circ} \mathrm{C}$ if LA-REIMS analysis is performed, either continuously or discontinuously, for several days. Consider aliquoting of the QC-pool for storage, in order to avoid freeze-thaw cycle effects.

334 LA-REIMS system set-up Laser set-up

MID-IR laser: The installation used comprises a MID infrared laser system (Opolette ${ }^{\mathrm{TM}}$ 337 HE2940, OPOTEK LLC, USA) consisting of a Q-switched Nd:YAG laser pumping Optical Parametric Oscillator (OPO). The system output is fixed at $2.94 \mu \mathrm{m}$ wavelength, $6 \mathrm{~mJ}$ peak

339 OPO energy, $7 \mathrm{~ns}$ pulse duration and $20 \mathrm{~Hz}$ repetition rate. Q-switch delay time is a tunable parameter with a direct impact on the transferred laser energy. The delay time may be optimized for each biofluid matrix separately depending on the produced peak intensity and repeatability, ranging between 165 and $180 \mu$ s (see 'Equipment set-up' section). The OPO beam transmission and focusing on the sampling area is achieved by the free space steering optics consisting of 3 metallic-coated mirrors and a plano-convex lens placed directly above the sampling surface. The sampling area position $5.5 \mathrm{~cm}$ below the focusing lens as well as

346 the laser focusing optics represent a fixed construction and, once set and aligned, do not 347 require routine tuning before each experiment. Laser beam and external optics alignment 348 verification, however, have to be performed on any new installation and systematically checked (Box 2).

$351 \mathrm{CO}_{2}$ laser: This is typically a modified surgical $\mathrm{CO}_{2}$ laser, operated at a $10.6 \mu \mathrm{m}$ wavelength.

352 Previous publications have utilised an OmniGuide FELS-25A (OmniGuide, Massachusetts, 
USA) or Aesculight VetScalpal (Aesculight, Washington, USA) using either mirror optics or a

354 hollow core fibre for laser beam transmission. A single mirror focusing handpiece is used to

355 direct the beam to a spot size of ca. $500 \mu \mathrm{m}$. These surgical $\mathrm{CO}_{2}$ lasers are used as they have

356 been created for the cutting and cauterising of animal tissue, and their wavelength and

357 absorbance profiles are thus complementary to the analysis of biological materials. They are

358 typically at the lower end of $\mathrm{CO}_{2}$ laser powers $(<10 \mathrm{~W})$ which are commercially available,

359 particularly when compared to industrial $\mathrm{CO}_{2}$ lasers ( $>100 \mathrm{~W}$ ) used for cutting/engraving

360 applications. Optimisation of $\mathrm{CO}_{2}$ laser power and delivery can be carried out for different

361 biological materials by altering the power output, pulsatile window, and distance between

362 focusing lens and sample. The output of the $\mathrm{CO}_{2}$ laser should be periodically checked with an

363 appropriate power monitor (such as Pronto-250-PLUS, Gentec Electro Optics, Quebec,

364 Canada) to ensure output is within the expected range.

BOX 2 | MID-IR laser alignment verification procedures • Timing 20-30 min

This box describes laser alignment and routine alignment verification for the MID-IR OPO laser. Alignment of the $\mathrm{CO}_{2}$ laser follows a similar principle.

! CAUTION Always wear eye protection with sufficient optical density while operating the laser. Consult Class 4 laser safety measures and equipment manual for additional precautions while operating the installation.

\section{Additional equipment}


- MIR Detector Cards: 1.5 to > $13.2 \mu \mathrm{m}$ (Thorlabs GmbH, Germany)

- Optical alignment target (shipped with Opolette ${ }^{T M}$ HE2940 MID-IR laser system)

\section{Procedure}

\section{Laser alignment verification}

$\triangle$ CRITICAL Beam alignment has to be performed on a new installation and assessed periodically following manufacturer recommendations or whenever laser misalignment is suspected (e.g. you have moved the setup).

1. On the front panel of the Opolette laser head, expose the laser pump port by moving the shutter marked '1064' to the side.

2. Screw the optical alignment target to the port with the uncovered area up (thus allowing visualization of the alignment target interior).

3. Set Q-switch delay time to $320 \mu$ s in the Q-switch configuration menu within laser control software or on handheld remote controller panel.

4. Activate the laser beam by consecutively pressing the 'Start' buttons on the flash lamp and Q-switch sections of the remote control/control software. The green light should appear around the alignment target aperture.

5. Check the position of the green light beam on the alignment target aperture. If the beam is centred, the laser is aligned and can be safely operated. Otherwise contact OPOTEK support centre.

6. Press the 'Stop' button subsequently on the flash lamp and Q-switch sections of the remote control/control software to deactivate the laser. 


\section{? TROUBLESHOOTING}

! CAUTION Do not decrease Q-switch delay time below $300 \mu$ s while aligning as it may damage the alignment verification target.

! CAUTION Do not further operate the system if the laser is misaligned or if the correct alignment cannot be verified.

\section{External optics alignment verification}

$\triangle$ CRITICAL Correct alignment of the laser steering optics is required for sufficient energy transmission and is crucial for operator safety.

7. To activate the alignment diode, toggle the switch at the back of the laser pump case.

The red light will become visible as projected on the sampling surface guided by the set of metallic-coated mirrors.

8. Check the projected position of the diode light on the working surface: the beam should pass through the middle of the focusing plano-convex lens. If not, adjust the positions of the metallic-coated mirrors.

9. Set the laser system in the operation mode as described in step 2 of the protocol.

10. Place the thermosensitive MIR detector card underneath the focusing lens so that the projected alignment diode light is visible on the card's surface.

11. Activate the laser beam by pressing the 'Start' button subsequently on the flash lamp and Q-switch sections of the remote control/control software.

12. The area of the discoloration on the MIR detector card will indicate the projection of the laser beam and that position should overlap with the alignment diode projection.

13. Deactivate the laser by consecutively pressing the 'Stop' buttons on the Q-switch and 
flash lamp sections of the remote control/control software.

14. Toggle the switch at the back of the laser pump case to deactivate the alignment diode.

\section{LA-REIMS sample analysis}

As a measure to ensure accurate laser energy transmission to the sample, it is essential to establish the correct position of the laser optics and/or sampling area prior to analysis in such a way that the laser focus point lays within the surface or the top layer of the specimen.

Besides, as the laser focusing lens is positioned merely $5 \mathrm{~cm}$ above the sampling surface and hence prone to contamination resulting from the ablation process, it is important to monitor and assure its cleanliness during the analytical runs. In particular, the state of the lens should

374 be evaluated every 5 to 10 ablations ${ }^{4}$, depending on the sample type. When contaminated,

375 the lens should be cleaned with a cotton swab drenched in ethanol. Interestingly, 376 positioning samples in a microtiter plate (automated sampling, see below) instead of on 377 glass microscope slides (manual sampling, see below) reduces the distribution of liquid 378 splashes during ablation and the resulting focusing lens contamination, hence the frequency 379 of cleaning. In case of automated sampling using microtiter plates significant focusing lens contamination and associated decrease in MS signal intensity (with more than one order)

381 has been observed only after 30 to 50 ablations (depending on the matrix type under study).

382 Nevertheless, we recommend lens cleaning to be performed at least every 5 ablations 383 during manual sampling and every 12 ablations during automated sampling to anticipate any possible signal issues (Step 30 of the protocol).

For the manual analysis, 20 to $50 \mu \mathrm{L}$ of the liquid biofluid (or thin layer of fecal blend) is pipetted on a glass microscope slide. Next, the slide is manually transferred under the laser 
beam focusing point and irradiated for $3 \mathrm{~s}$ and removed. In between sample ablation, the

solvent signal is recorded for 3 to $4 \mathrm{~s}$ to ensure that the signal intensity drops to the background level and guarantee good peak resolution (Fig. 3). The method acquisition time

390 is set to $30 \mathrm{~s}$ per sample which is sufficient to generate 2 to 3 technical replicates (ablations)

391 within one acquisition period, if desired ${ }^{12}$.

392 The automated sampling platform employs a simple in-house made two-axis (XY) motorised 393 precision position stage (Fig. 4a) that can hold up to 6 different 96 -well plates on the mobile 394 platform. The customized controlling software defines the platform movements and laser 395 activation/deactivation sequence. The moving stage subsequently positions the samples in the separate wells under the laser focusing point. Every irradiation (ablation) is followed by a 397 cooldown period during which the next well is positioned below the laser focusing point and a new acquisition datafile is initiated by the recording software. The sample volume within each well is recommended to be at least 50 and up to $100 \mu \mathrm{L}$ as bigger volumes are better preserved during prolonged exposure of the liquid samples to ambient conditions during automated analysis as opposed to manual sampling. Although the recommended sample volume is substantially higher when using automated sampling compared to manual sampling, the (semi-)liquid within the well plates can be subjected to repeated laser 404 ablations without loss of signal intensity or spectrum quality (Table 3). An additional measure that can be taken to minimize passive evaporation of the specimens within the wells during analysis, is the installation of a local thermoelectric (Peltier) cooling element in 407 the sampling area to create an optimal (cooled) microenvironment.

408 Daily calibrations of the MS instrument during active sample analysis periods should be 409 performed according to manufacturer guidelines to ensure the highest mass accuracy (e.g. sodium formate solution in 90:10 isopropylalcohol:water $(\mathrm{v} / \mathrm{v})$ for calibration of Waters 
411 QToF/Q-IMS-ToF instruments) ${ }^{7,12}$. Moreover, when starting the instrument after cleaning or

412 planning sample analysis for different matrices, it is advised to run 10 to 20 pooled eQC

413 samples in both ion modes to secure adequate instrument conditioning before starting the

414 analysis or switching to another matrix type $\mathrm{e}^{12}$. This measure reduces initial signal drift within

415 the samples of interest during the actual experiment. A representative number of iQC

416 samples (two between every 20 to 40 analytical run) should be analysed to monitor and

417 compensate for possible MS signal output changes during the analytical run (analytical drift).

418 A final strategy to ensure the quality of collected data is the usage of external standards

419 infused together with the solvent matrix (e.g. leucine-enkephalin ${ }^{7}$ or a combination of

420 palmitic acid- $d_{31}$ and 1,2-dimyristoy- $d_{54}$-sn-glycero-3-phosphocholine ${ }^{12}$ ). This allows for

421 evaluation of instrument analytical performance as well as lock mass correction and data

422 normalization.

423

424 LA-REIMS data collection

425 Data collection is performed using MS vendor-specific software. All MS methods for

426 automated programs are set at $18 \mathrm{~s}$ per sample acquisition time, while $30 \mathrm{~s}$ is used upon

427 manual sampling ${ }^{4}$. Mass spectra can be collected as one raw data file per sample, which may

428 reduce downstream data processing but increase time for data collection. Alternatively, one

429 raw data file per microwell plate or batch could be generated, which may then decrease

430 time for data collection as a result of reduced instrument electronic control but requires the

431 splitting of files and thus increases subsequent downstream data processing.

432

433 Data analysis \& classification modelling 
434 Data analysis can be performed using any kind of software that runs the appropriate sort of 435 algorithms. Box 3 describes the procedures that were followed, using the software that was 436 available to the authors of this protocol.

437 During LA-REIMS analysis, molecular fingerprints are acquired by MS vendor-specific 438 software, such as MassLynx ${ }^{\mathrm{TM}}$ (v4.2, Waters, stored as .raw directories) in case of Waters 439 instruments. Prior to performing classification modelling, several pre-processing steps are 440 implemented to select representative burns, subtract background and noise, and perform 441 peak picking (Box 3, Steps 1-12). In general, these pre-processing steps are also executed 442 using vendor-specific software (Progenesis ${ }^{\circledR} \quad$ Bridge, Progenesis $^{\circledR}$ QI, AMX or Live ID, 443 Waters). Alternatively, spectra can be pre-processed using freely available open-source 444 metabolomics software such as $\mathrm{XCMS}^{39}, \mathrm{MZmine}^{40}$, etc.

445 Following pre-processing, peak lists of $\mathrm{m} / \mathrm{z}$-features are subjected to multivariate statistical 446 analysis, typically including principal component analysis $\left(\mathrm{PCA}^{41}\right)$ and orthogonal partial least 447 squares discriminant analysis (OPLS-DA ${ }^{42}$ ) (using software packages such as SIMCA (v15, 448 Sartorius)) (Box 3, Steps 13-22). PCA serves as an unsupervised dimension reduction 449 technique to investigate variance within the dataset. OPLS-DA is a supervised regression 450 methodology whereby user-defined groups are taken into account, thereby pursuing 451 classification of the groups based on discriminative features. The (validated) models can be 452 utilized to extract discriminative features that can further undergo identification using 453 fragmentation experiments and database matching.

454 In Box 3, data analysis and classification modelling from raw files collected on the LA-REIMS 455 instrument (Waters), is described by the sequential use of Progenesis ${ }^{\circledR}$ Bridge (v1.0.29, 456 Waters), Progenesis ${ }^{\circledR}$ QI (v2.1, Waters) and SIMCA (v15, Sartorius) software. 


\section{BOX 3 | LA-REIMS data analysis \& classification modelling • Timing $1 \mathrm{~h}$ for ca. 500}

samples

This box describes the pre-processing and classification modelling (steps 31-34 of the Procedure) that is performed after data acquisition by LA-REIMS. This biostatistical pipeline can be used for any type of analyte described in this protocol, and uses three commercially available software packages.

The first program, Progenesis ${ }^{\circledR}$ Bridge (v1.0.29, Waters), is capable of handling Waters MS .raw directories and performs background subtraction, lock mass correction (optional), creates extracted ion chromatograms for each burn and separates multiple burns in each raw folder into individual raw folders. This separation enables individual burns to be treated as individual samples. For further treatment in Progenesis ${ }^{\circledR} \mathrm{QI}$, all individual peak raw folders can be loaded or 1 peak per sample can be selected. In case of the latter, it is suggested to use the peak with the highest total ion current (TIC) value. TIC can be assessed by manually inspecting each chromatogram in MassLynx ${ }^{\mathrm{TM}}$ (Waters).

Next, the raw data are ready for importing into the second software program, Progenesis $^{\circledR}$ QI (v2.1, Waters), where peak picking and TIC normalization is performed and lists of $m / z$-features are generated. In a third software program, SIMCA ( $v 15$, Sartorius), fingerprints can be visualised, quantified and statistically classified, and discriminating features can be defined. The data analysis pipeline consists of the steps summed below.

\section{Procedure}


1. Open Progenesis ${ }^{\circledR}$ Bridge, fill in fields a. to d., as described below, and click 'Run'.

a. Source: directory path containing non-processed Waters .raw directories.

b. Output path: directory path where processed data will be stored.

c. TIC replicate threshold: threshold between background and peak intensity. Should be set at approximately $1 / 3$ of peak height.

\section{? TROUBLESHOOTING}

d. Lock Mass Correction: None.

2. Open Progenesis ${ }^{\circledR}$ QI, click 'File' > 'New' and fill in experiment name and directory path.

3. Set the analysis parameters of new experiment a. to c., as described below, and click 'Next'.

a. Type of machine: direct sample analysis.

b. Data format: profile data.

c. Ionization polarity: select correct polarity according to the experiment.

4. Select the possible adducts according to the experiment and click 'Create experiment'.

5. In the created experiment window, in tab 'Import Data', choose Waters (.raw) format and click 'Import' to select and import files that need to be processed.

6. In the created experiment window, in tab 'Import Data', click 'Start automatic processing'.

a. Experimental design can be left empty at this stage, click 'Next'.

b. Set the peak picking parameters by clicking 'Set parameters', fill in and click 'OK'. 
i. Peak picking limits > Sensitivity: Automatic - default.

ii. For the other parameters, default values should be attained.

7. In the created experiment window, in tab 'Experimental Design Setup', click 'Between-subject Design', fill in and click 'Create design'.

a. Design: new design.

b. Group runs manually.

c. Create design.

8. In the created experiment window, in tab 'Experimental Design Setup', select all samples, click 'Add Selected Runs to Condition'.

9. In the created experiment window, in tab 'Peak Picking', click 'Review Normalisation', click 'Normalisation Method' and select 'Normalize using total ion abundance'.

10. In the created experiment window, in tab 'Review Compounds', check information of compounds.

11. Click 'File' > 'Export compounds measurements', select information to export and click 'OK'.

a. Select Compound, $m / z$, normalized abundance, raw abundance.

12. Transpose, rearrange and add classification labels prior to loading peak lists into SIMCA. Optionally, (internal) quality control normalization can be performed during this step.

13. Open SIMCA, click 'File' > 'New' > 'Regular Project'.

14. Paste the data from the .csv file into the 'SIMCA Import' window.

15. Create workset by defining the data, click 'Finish import'. 

a. Define the row head as 'Primary ID'.
b. Define the column head as 'Primary ID'.
c. Define the $2^{\text {nd }}$ column head to select a data type ' $Y$ variable with Qualitative Data' or ' $Y$ with Quantitative Data'; depending on the experiment type.

\section{? TROUBLESHOOTING}

16. In the 'Home' tab, click 'Change model type' > 'PCA-X model'.

17. In the 'Home' tab, Click 'Edit' to control the PCA settings, click 'OK'.

a. Click tab 'Scale', select all and choose type: e.g. Par (Pareto scaling).

b. Click tab 'Transform', select all, choose 'Specify transformation': Log, click 'Set', select box 'If one variable in the $\mathrm{X}$ or $\mathrm{Y}$ block needs transformation, transform all selected variables in that block' and click 'Transform'.

18. In the 'Home' tab, Click 'Autofit' and 'Scores' to visualize model.

19. In the 'Home' tab, Click 'New as' > 'PCA-X' (copy settings previous model), click 'OK'.

20. In the 'Home' tab, click 'Change model type' > 'OPLS-DA model'.

21. In the 'Home' tab, Click 'Edit' to control the PCA settings, click 'OK'.

a. Click tab 'Observations' to check classes are selected; if not: select samples per class, name the new class and click 'Set'.

b. Click tab 'Observations' to exclude samples (e.g. QCs): select sample and click 'Exclude'.

22. In the 'Home' tab, Click 'Autofit' and 'Scores' to visualize model. 
- Stool samples: For development of the LA-REIMS procedures as well as to perform rapid fingerprinting in a context of clinical diagnostics, stool samples were collected from study participants that provided written, informed consent, following approval of the study by the ethics committee. For the examples described in this protocol, samples were collected under University Hospital Ghent Ethical Committee (EC 2016/0673). Collection of stool material is preferable performed by using specifically designed equipment, such as e.g. the Fecotainer ${ }^{\circledR}$ or FecesCatcher (De Fecesvanger, The Netherlands; both of these products provide detailed instructions. Other tools (e.g. plastic bag, plastic disposable, bucket, etc.) are also suitable although considered less convenient for the study participant. Upon collection, fresh stool samples should immediately be frozen at $-80{ }^{\circ} \mathrm{C}$ if possible, i.e. if sample collection takes place at e.g. the hospital, research facility, etc. If the sample is collected at the participant's home, temporary storage at $-20{ }^{\circ} \mathrm{C}$ will be needed, but should be kept as short as possible. Transport of the sample to the relevant facilities should be realized under cooled conditions $\left(0{ }^{\circ} \mathrm{C}\right.$ or lower; using ice packs, dry ice, or liquid nitrogen). Prior to storage at $-80^{\circ} \mathrm{C}$, lyophilization is advised in order to remove water and stop microbial activity. In this study, lyophilization was achieved by placing the samples in a Christ 1-4 Alpha LSCplus lyophilisator for $48 \pm 4 \mathrm{~h}$ at $-40{ }^{\circ} \mathrm{C}$. Lyophilized samples were grinded with pestle and mortar and sieved (mesh size of 2.5 $\mathrm{mm}$ ) to obtain a homogenous powder.

To demonstrate the application potential of LA-REIMS stool fingerprinting in a clinical 481 context, stool material was collected from male and female study participants with normal blood glucose levels and those that were diagnosed with type 2 diabetes, 
thereby applying a classification threshold of $60 \mathrm{mmol} / \mathrm{mol}$ glycated haemoglobin $(\mathrm{Hb}$ $484 \quad$ A1c).

$485<$ CRITICAL $>$ It is important that stool material is not contaminated with urine during its 486 collection. <CRITICAL> Fresh (non-lyophilized) samples should be kept frozen at all times $487 \quad$ to avoid metabolic alterations.

- Blood samples (plasma): For development of the LA-REIMS procedures as well as to 489 perform rapid fingerprinting in a context of clinical diagnostics, blood samples were 490 collected from study participants that provided written, informed consent, following 491 approval of the study by the ethics committee. For the examples, described in this 492 protocol, samples were collected under University Hospital Ghent Ethical Committee (EC $493 \quad$ 2016/0673).

494 Blood samples were collected from the study participants using a $10-\mathrm{mL}$ syringe and 495 transferred into a heparin-containing tube, which was centrifuged for $10 \mathrm{~min}$ at 2800 to $4963000 \times \mathrm{g}\left(\right.$ at $\left.2-8^{\circ} \mathrm{C}\right)$. The obtained plasma was transferred to a cryovial and stored at $49780{ }^{\circ} \mathrm{C}$. If needed, transport of the plasma samples should be realized under cooled 498 conditions $\left(0{ }^{\circ} \mathrm{C}\right.$ or lower; using ice packs, dry ice, or liquid nitrogen). <CRITICAL> 499 Samples should be kept frozen at all times to avoid metabolic alterations. Thawing of the 500 material for LA-REIMS analysis should be performed at $4-6{ }^{\circ} \mathrm{C}$. <CRITICAL> Upon long501 term storage ( $>1$ year), protein precipitation may occur, for which additional purification 502 measures should be taken prior to LA-REIMS analysis.

- Saliva samples: For development of the LA-REIMS procedures as well as to perform rapid

504 fingerprinting in a context of clinical diagnostics, saliva samples were collected from 505 study participants that provided written, informed consent (or parents in case of 506 minors), following approval of the study by the ethics committee. For the examples, 
described in this protocol, samples were collected under University Hospital Ghent

$508 \quad$ Ethical Committee (EC 2016/0673).

509 Collection of saliva should be established under standardized protocols to minimize the

510 impact of confounding factors; e.g. with respect to brushing of the teeth, the time slot of

511 sample collection, dietary restrictions with respect to food and drinks consumption prior

512 to sample collection, etc. Collection of saliva can be achieved by passive drooling or

513 usage of suitable swabs such as e.g. Salivette ${ }^{\circledR}$ synthetic (Sarstedt, Germany) or SalivaBio

514 (SalivaBio, Germany) swabs. After collecting the saliva material in a cryovial, snap-

515 freezing and/or storage at $-80{ }^{\circ} \mathrm{C}$ is designated. Given the nature of the sample

516 collection, it is possible and advised to collect samples at equipped facilities where these

517 protocols can be applied to minimize metabolic alterations which may occur during e.g.

$518 \quad$ sample transport.

519 To demonstrate the application potential of LA-REIMS saliva fingerprinting in a clinical 520 context, saliva material was collected from children and adolescents (6 to 16 years),

521 which were classified according to weight status, using the IOTF-score (z-score for BMI)

522 (IOTF of 0 for normal weight, 1 for overweight, and 2 for obesity).

523 <CRITICAL> Fresh samples should be kept frozen at all times to avoid metabolic

524 alterations. Thawing of the material for LA-REIMS analysis should be performed at $4-6{ }^{\circ} \mathrm{C}$.

525 - Urine samples: For development of the LA-REIMS procedures as well as to perform rapid

526 fingerprinting in a context of clinical diagnostics, urine samples were collected from

527 study participants that provided written, informed consent (or parents in case of

528 minors), following approval of the study by the ethics committee. For the examples,

529 described in this protocol, samples were collected under University Hospital Ghent

$530 \quad$ Ethical Committee (EC 2017/1634). 
531 Urine samples can be collected in a plastic $100-\mathrm{mL}$ container in case of adults or children

532 that are neat, whereas alternative tools need to be used in case that children are not

533 neat; e.g. Sterisets Newcastle Urine Collection Packs (Sterisets Medical Devices,

534 Portugal). With respect to the latter, urine can be extracted from these packs using a

535 syringe. Samples should be stored as soon as possible at $-20^{\circ} \mathrm{C}$ (at the participant's

536 home) or $-80{ }^{\circ} \mathrm{C}$ (at equipped facilities). If transport is needed, this should be realized

537 under cooled conditions $\left(0{ }^{\circ} \mathrm{C}\right.$ or lower; using ice packs, dry ice, or liquid nitrogen).

$538<$ CRITICAL> It is important that urine is not contaminated with stool material when

539 collecting from children that are not neat. <CRITICAL $>$ Samples should be kept frozen at

540 all times to avoid metabolic alterations. Thawing of the material for LA-REIMS analysis

541 should be performed at $4-6^{\circ} \mathrm{C}$.

542 Sputum: For development of the LA-REIMS procedures, sputum samples were obtained

543 from the Royal Brompton Hospital as part of routine sample collection for monitoring of

544 cystic fibrosis patients. Sputum can be collected as either spontaneous or induced

545 dependent on clinical guidelines and practice in sterile universal containers. Samples can

546 be treated with Sputasol to allow easier handling or analysed neat. They can further be

547 analysed immediately upon sampling or stored at $-80^{\circ} \mathrm{C}$. <CRITICAI> Samples should be

548 kept frozen at all times to avoid metabolic alterations. Thawing of the material for LA-

549 REIMS analysis should be performed at 4-6 ${ }^{\circ} \mathrm{C}$. ! Caution For safety purposes, samples

550 should not be collected from patients with positive results or suspected symptoms of

551 tuberculosis, unless samples can be stored, processed, and analysed in Category III

552 biohazard facilities. If samples contain blood, they should not be analysed and should be

553 disposed of immediately. 
- Human milk: For development of LA-REIMS procedures, human milk samples were

555 collected from a cohort of breastfeeding dyads with nursling ages ranging between 3 to

55624 months. For this purpose, samples were collected under a sub-project (approval

557 number R18006) of the Breastmilk Epigenetic Cohort Study under the Imperial College

$558 \quad$ Healthcare Tissue Bank (HTA licence 12275), which received ethical approval from the

559 Wales Regional Ethics Committee (reference 17/WA/0161). Participants should be free

560 to choose method of expression (hand, electric, or manual pump) but sterility of

561 collection equipment (pump, tubing, containers) should be achieved through steam

562 sterilization following manufacturers' procedures. Samples should be collected into

563 sterile containers suited to the volume of collected human milk sample. Samples can be

564 analysed immediately (within $60 \mathrm{~min}$ of collection) or stored at $-20{ }^{\circ} \mathrm{C}$ or $-80{ }^{\circ} \mathrm{C}$

565 depending on availability of facilities. <CRITICAL> Samples should be kept frozen at all

566 times to avoid metabolic alterations. Thawing of the material for LA-REIMS analysis

567 should be performed at $4-6^{\circ} \mathrm{C}$.

568

\section{Reagents}

570 - Isopropylalcohol (LC-MS grade, Sigma Aldrich, cat. no. 34863)

571 - Ultrapure water $(0.055 \mu \mathrm{S} / \mathrm{cm}$, purchased or obtained from water-purification

$572 \quad$ systems such as Sartorius Arium 661 UV, Sartorius, Germany)

573 - Sodium formate calibration solution (see 'Reagent set-up' section)

574 - $0.5 \mathrm{mM}$ sodium hydroxide in 90:10 isopropylalcohol /water (v/v) (Waters, cat. no.

$575 \quad 186007052)$

576 - Formic acid > 99.0\% (LC-MS grade, Fisher Scientific, Sigma Aldrich, cat. no. 117-50)

577 - Methanol (LC-MS grade, Sigma Aldrich, cat. no. 646377) 
580 Depending on the experimental set-up, one or multiple compounds may be co-infused with

581 the isopropylalcohol solvent matrix during analysis. Details on how to prepare the solutions 582 are in the section 'Reagent set-up'.

583 - (Optional) leucine-enkephalin (Waters, cat. no. 186006013)

584 - (Optional) palmitic acid-d $d_{31}$ (Sigma Aldrich, cat. no. 366897)

585 - (Optional) 1,2-dimyristoyl-d ${ }_{54}$-sn-glycero-3-phosphocholine (Sigma Aldrich, cat. no.

$586 \quad 711047)$

587

588

Equipment

589

590

591

592

593

594

595

596

597

598

599

600

- $\quad$ Nitril gloves (EcoSHIELD ${ }^{\mathrm{TM}}$ Eco NitrilePF 250, NOVOLAB NV, Belgium)

- Laser Safety Glasses LG16 4.00, 41\% Visible Light Transmi Commodity: 9004 9010, RoHS: A (compliant 2011/65/EU) (Thorlabs GmbH, Germany)

\section{$\underline{\text { General lab equipment }}$}

- Wipe away ${ }^{\circledR}$ wipes

- Micropipettes (100-1000 $\mu \mathrm{L}$, Eppendorf, Germany)

- Volumetric beakers (VWR, Borosilicate glass 3.3, 100-250-600-1000 mL)

- Cotton cleaning swabs (Biolab, Hungary, cat. no. CTA90003)

- Microscope blank glass slides (Marienfeld, Germany, cat. no. 1000000)

- Microtiter plates (NOVOLAB NV, Belgium, cat. no. 32004)

- Elmasonic $\mathrm{P}^{\oplus}$ ultrasonic bath (Elma, Germany, cat. no. 1013737 )

601 $\underline{\text { Laser set-up }}$ 
602

603

$604 \quad$ separation optics and laser power supply

605 - Power meter (Quantel, USA)

606 - Aluminium breadboard $200 \times 200 \times 12.7 \mathrm{~mm}$ and M6 Taps (Thorlabs GmbH, $607 \quad$ Germany)

608 - Fee space optics, enfolding a line of metallic or gold-coated mirrors $609 \quad$ (OptoSigma Global Top, France)

610 - Fixed Lens holders LHG RoHS W4148 (Sigma-koki, Japan), kinematic mirror holders 611 RoHS W4002 (Sigma-koki, Japan)

612 - Pedestal bases $(45.8 \mathrm{~mm})$ onto which lenses and mirrors are mounted using pedestal 613 clamps RoHS W6039 (Sigma-koki, Japan)

614 - Clamps and damped rods for every mirror and/or lens (using a 65x65mm M6 615 plateau) (Sigma-koki, Japan)

616 - Z Axis Aluminium translation stages $(65 \times 65 \mathrm{~mm})$ (Sigma-koki, Japan)

617 - $\mathrm{CAF}_{2}$ Plano convex lens RoHS W3189 (Thorlabs GmbH, Germany)

618 - PEEK tubing (0.010 in. ID; $30 \mathrm{~cm}$, Thermo Fisher Scientific, USA, cat. no. 10574855)

619 - Cytiva PEEK Connectors 1/16 in. Luer (Thermo Fisher Scientific, USA, cat. no.

$620 \quad 11370232)$

$621 \quad \underline{\text { REIMS }}$

622 - Polytetrafluorethylene tubing (2.5 m, $3.2 \mathrm{~mm}$ O.D., $1.6 \mathrm{~mm}$ I.D., Sigma Aldrich, cat. 623 no. 

58699) connected to the REIMS interface by means of a T-shaped connector piece (prototype, Waters Research Center, Hungary)

- REIMS source (prototype, Waters Research Center, Hungary) with a $20 \mathrm{~cm}$ inlet capillary of $0.7 \mathrm{~mm}$ I.D. mounted onto the housing of the T-shaped connector piece

\section{Software}

637

2. Progenesis ${ }^{\circledR}$ Bridge (v1.0.29, Waters)

3. Progenesis ${ }^{\circledR} \mathrm{QI}$ (V2.1, Waters)

4. SIMCA (v15, Sartorius)

644 1. Prepare $1 \mathrm{~mL}$ of $10 \%$ formic acid/aqueous solution by mixing $900 \mu \mathrm{L}$ of ultrapure water and $100 \mu \mathrm{L}$ LC-MS grade formic acid; sonicate for $5 \min (40 \mathrm{kHz}$, fixed 646 frequency, no heating). 
2. Mix $100 \mu \mathrm{L}$ of $10 \%$ formic acid/aqueous solution to $30 \mathrm{~mL}$ of $0.5 \mathrm{mM}$ sodium hydroxide in 90:10 isopropylalcohol/water (v/v) and transfer to an amber glass vial; sonicate for $5 \min (40 \mathrm{kHz}$, fixed frequency, no heating).

PAUSE POINT The sodium formate solution can be stored at $4{ }^{\circ} \mathrm{C}$ for up to 3 months.

\section{(optional) Leucine-enkephalin in isopropylalcohol}

Can be used as an external standard for analysis in both positive and negative ionization mode ${ }^{7}$.

1. Add $7.5 \mathrm{~mL}$ of ultrapure water to a $3 \mathrm{mg}$ bottle of leucine-enkephalin.

2. Recap, vortex for $30 \mathrm{~s}$ at $14 \mathrm{xg}$ and sonicate for $5 \mathrm{~min}$; the resulting $400 \mathrm{ng} / \mathrm{\mu L}$ solution can be stored at $-20^{\circ} \mathrm{C}$ for up to 3 months.

3. Transfer $5 \mu \mathrm{L}$ of $400 \mathrm{ng} / \mu \mathrm{L}$ leucine-enkephalin solution to a $50 \mathrm{~mL}$ glass vial, add $20 \mathrm{~mL}$ of isopropylalcohol to obtain a $0.1 \mathrm{ng} / \mu \mathrm{L}$ stock solution. Sonicate for 5 minutes (40 kHz, fixed frequency, no heating). Label and store at $-20{ }^{\circ} \mathrm{C}$ for up to one month.

4. Transfer $1 \mathrm{~mL}$ of $0.1 \mathrm{ng} / \mu \mathrm{L}$ leucine-enkephalin solution to $9 \mathrm{~mL}$ of isopropylalcohol stored in an amber glass vial to obtain a $10 \mathrm{ng} / \mathrm{mL}$ solution; sonicate for $5 \min (40 \mathrm{kHz}$, fixed frequency, no heating).

- PAUSE POINT The resulting solution can be used directly in the experiment or stored at $-20^{\circ} \mathrm{C}$ for up to one week.

\section{(optional) Palmitic acid-d $d_{31}$ in isopropylalcohol}

Can be used as external standard for negative ionization only. In case of alternating between positive and negative ionization mode during LA-REIMS analysis, it is possible to add 1,2- 
671 dimyristoyl-d $d_{54}$-sn-glycero-3-phosphocholine to this solution to also cover positive

672 ionization $^{13}$.

673 1. Weigh $1 \mathrm{mg}$ of palmitic acid- $\mathrm{d}_{31}$ into a $5 \mathrm{~mL}$ glass vial.

674 2. Add $1 \mathrm{~mL}$ of ultrapure water, vortex for $30 \mathrm{~s}$ at $14 \mathrm{xg}$ and sonicate for $5 \mathrm{~min}$ (40

$675 \mathrm{kHz}$, fixed frequency, no heating); the resulting $1 \mathrm{mg} / \mathrm{mL}$ stock solution can be

$676 \quad$ stored at $-20^{\circ} \mathrm{C}$ up to 3 months.

677 3. Transfer $50 \mu \mathrm{L}$ of $1 \mathrm{mg} / \mathrm{mL}$ stock solution into a $10 \mathrm{~mL}$ glass bottle, add $9.95 \mathrm{~mL}$ of

678 isopropylalcohol; sonicate for $5 \min (40 \mathrm{kHz}$, fixed frequency, no heating).

679 - PAUSE POINT The resulting $5 \mathrm{ng} / \mu \mathrm{L}$ solution can be used for sample analysis or stored 680 at $-20^{\circ} \mathrm{C}$ for up to one month.

681

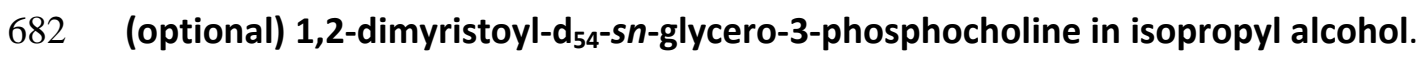

683 Can be used as external standard for positive ionization only. In case of alternating between 684 positive and negative ionization mode during LA-REIMS analysis, it is possible to add palmitic 685 acid- $d_{31}$ to this solution to also cover negative ionization ${ }^{13}$.

686

687 1. Weigh $10 \mathrm{mg}$ of 1,2-dimyristoyl- $\mathrm{d}_{54}$-sn-glycero-3-phosphocholine in a $10 \mathrm{~mL}$ glass $688 \quad$ vial.

689 2. Add $10 \mathrm{~mL}$ of methanol, vortex for $30 \mathrm{~s}$ at $14 \mathrm{xg}$ and sonicate for $5 \mathrm{~min}(40 \mathrm{kHz}$, 690 fixed frequency, no heating); the resulting $1 \mathrm{mg} / \mathrm{mL}$ stock solution can be stored $691 \quad$ at $-20^{\circ} \mathrm{C}$ up to 3 months.

692 3. Transfer $100 \mu \mathrm{L}$ of $1 \mathrm{mg} / \mathrm{mL}$ stock solution into a $10 \mathrm{~mL}$ glass bottle, add $9.9 \mathrm{~mL}$ of 693 isopropylalcohol; sonicate for $5 \mathrm{~min}(40 \mathrm{kHz}$, fixed frequency, no heating). 

stored at $-20^{\circ} \mathrm{C}$ for up to one month.

696

697

Equipment set-up

698

MS parameters

699 Use the following general settings for the Waters Xevo G2-XS QToF instrument.

\section{Instrument parameters}

\begin{tabular}{l|l}
\hline Mass range, Da & $50-1200$ \\
Analyser mode & Sensitivity \\
\hline Data format & Continuum \\
\hline Acquisition start time, min & 0 \\
\hline Acquisition end time, min & 0.5 (manual sampling); 0.3 (automated sampling) \\
\hline Target enhancement & Off
\end{tabular}

701 Use the following optimized set-up parameters for the Waters Xevo G2-XS QToF instrument 702 combined with MID-IR laser (Q-switch delay time) or $\mathrm{CO}_{2}$ laser (laser power and pulse 703 values) for the specified biofluids in positive $(+)$ and negative $(-)$ ion polarity modes. 704 Abbreviations used; NA (not applicable).

\begin{tabular}{ccccccc} 
& Urine & Feces & Saliva & Plasma & Sputum & Human Milk \\
\cline { 2 - 6 } Polarity & $+/-$ & $+/-$ & $+/-$ & $+/-$ & $+/-$ & $+/-$ \\
\hline Solvent flow & 0.30 & 0.15 & $0.12 / 0.20$ & $0.30 / 0.20$ & 0.25 & 0.25 \\
rate, $\mathrm{mL} / \mathrm{min}$ & & & & & & \\
Cone voltage, $\mathrm{V}$ & 25 & $40 / 70$ & $20 / 45$ & 30 & 80 & 80
\end{tabular}




\begin{tabular}{|c|c|c|c|c|c|c|}
\hline $\begin{array}{l}\text { Heater bias } \\
\text { voltage, } \mathrm{V}\end{array}$ & 25 & 40 & 75 / 70 & 40 & 50 & 50 \\
\hline $\begin{array}{l}\text { Laser wave- } \\
\text { length, } \mu \mathrm{m}\end{array}$ & 2.94 & 2.94 & 2.94 & 2.94 & 10.64 & 10.64 \\
\hline $\begin{array}{l}\text { Laser Q-switch } \\
\text { delay time, } \mu \mathrm{s}\end{array}$ & 185 & 180 & 165 / 180 & 170 / 165 & NA & NA \\
\hline Laser power, W & NA & NA & NA & NA & 2.0 & 3.5 \\
\hline Pulse time, ms & NA & NA & NA & NA & 40 & 40 \\
\hline Scan time, s & $0.7 / 0.5$ & 0.3 & 0.5 & 0.7 & 1.0 & 1.0 \\
\hline
\end{tabular}

705

Procedure

Set-up of the sample holder or container $\bullet$ Timing 1-2 min per sample

708

1. Transfer the samples to an appropriate sample holder or container. Sample handling will be different depending on the adopted sampling approach (manual or automated) as well as on the sample matrix (liquid or semi-fluid). For manual sampling of liquids and semi-fluids ( $A$ and $B$ ) multiple sample slides can be prepared in advance, but preferably not more than 10 samples at a time, as the thin sample layer will dry-out within 20 to 30 min under ambient conditions. For automated sampling, the position of the samples within the 96-well plate corresponds to the order within the acquisition sequence ( $\mathrm{C}$ and D). If more than one well plate is to be analysed, it is recommended to cover the well plates with a self-adhesive polyester foil to reduce sample evaporation pending analysis.

\section{A. Manual sampling of liquids}

Using a $100 \mu \mathrm{L}$ pipet, transfer 20 to $50 \mu \mathrm{L}$ of liquid sample (e.g. plasma, urine, saliva, human milk, sputum treated with Sputasol) to the glass microscope slide. Use separate slides for 


\section{B. Manual sampling of semi-fluids}

722 Using a disposable sterile spatula, apply a thin layer (approximately 50-80 mg) of semi-fluid

723 sample (e.g. feces, untreated sputum) to the microscope slide and distribute evenly.

724

725

\section{Automated sampling of liquids}

Using a $100 \mu \mathrm{L}$ pipet, transfer $100 \mu \mathrm{L}$ of liquid sample (e.g. plasma, urine, saliva, human milk, sputum treated with Sputasol) to its designated well within a 96-well plate.

\section{Automated sampling of semi-fluids}

Using a disposable sterile spatula, fill the sample well with semi-fluid sample (e.g. feces, untreated sputum) to approximately half of the well's height (80 to $100 \mathrm{mg}$ of sample generally suffices).

\section{Set-up of the laser $\bullet$ Timing $10-15 \mathrm{~min}$}

2. The authors of this protocol had access to an Nd:YAG MIR OPO laser and $\mathrm{CO}_{2}$ laser for their experiments. Both lasers have been demonstrated suitable to achieve ablation of liquid and semi-fluid biological material. Preparation of the lasers for sample ablation is detailed in option A and option B, respectively.

\section{A. MIR OPO laser}

(i) On the front panel of the laser pump power supply, turn the safety key to the 'On' position ( $45^{\circ}$ clockwise).

(ii) The LED indicators within the water reservoir level window will light up, ensure that the water level is above the window minimum indication. 
(iii) In the system menu of the laser control panel, choose 'system settings' and check the

744 coolant temperature readback. Wait approximately 5 to $10 \mathrm{~min}$ until the coolant $745 \quad$ temperature rises up to $37.5^{\circ} \mathrm{C}$.

746 (iv) In the Q-switch configuration menu of the laser control panel, set the Q-switch delay

747 time to the optimized value for the selected matrix ('Equipment set-up’ section).

748 (v) In the Q-switch configuration menu, set the Q-switch repetition rate to F/01 (20 Hz).

749 This value is standard for all described methods independent of the matrix.

750

751

752

753

754

755

756

757

758

759

760

761

762

763

764

\section{B. $\mathrm{CO}_{2}$ laser}

(i) Ensure that the laser hollow core fibre is connected correctly to both the laser unit and the focusing system and that there is no visible damage to the fibre or connecting points.

(ii) Turn on the front panel of the laser and ensure that any connecting gas supplies for cooling are turned on to the correct pressure setting.

(iii) The laser will run through a self-check procedure to ensure it is operating at the appropriate level. Any errors will be communicated on the display screen for user troubleshooting.

(iv) With the laser on 'Standby' mode, adjust the laser operating settings to the required pulsatile mode, pulsatile duration, output power, and gas flow pressure.

(v) On the display screen, switch the laser to 'On' mode and wait $10 \mathrm{~s}$ to ensure no system error messages appear.

Connecting REIMS sampling tube, solvent line and MS source • Timing 5-10 min 
3. Fix one end of the PTFE transfer tube $1 \mathrm{~cm}$ above and $3-4 \mathrm{~mm}$ to the side of the laser focus point on the sampling surface (to check the position of the laser focus point, follow steps 2.1 to 2.8. as described in Box 2).

4. Using PEEK fitting (1/4-28 flangeless nuts for $1 / 8^{\prime \prime}$ OD tubing), connect the other end of the PTFE transfer tube to the T-piece placed at the end of the ion transfer capillary in the REIMS source (Fig. 4b).

5. Fill the $10 \mathrm{~mL}$ glass Luer-lock syringe with isopropylalcohol (for isopropylalcohol containing reference external standards follow 'Reagent set-up' section).

6. Use Cytiva Luer PEEK Connectors to connect the syringe to the PEEK tubing; use twopiece PEEK fittings with ferrules to connect the solvent live with the T-piece at the level of the MS source (Fig. 4b).

776

System preparation and optimization • Timing 20-25 min

7. On the front panel of the solvent pump, set the solvent flow rate according to the

9. (Optional) If using external standards within the isopropylalcohol solution, check if the

8. Observe the MS signal from the solvent flow on the MassLynx ${ }^{T M}$ tune page. Record the blank solvent signal and compare the spectrum profile with the blank solvent signal from a previous experiment.

\section{? TROUBLESHOOTING} $\mathrm{m} / \mathrm{z}$-peaks of these external standards are clearly visible within the solvent spectrum on the MassLynx ${ }^{\mathrm{TM}}$ tune page. 
10. Follow the procedure described in the 'Sample analysis' section (step $30 \mathrm{~A}$ and B), analyse 10 eQC samples, respectively, in negative and positive ion mode. This additional measure will ensure conditioning of the instrument with the matrix-specific ions and reduce MS signal variation over time during subsequent analysis of biological samples.

External calibration of the REIMS • Timing $40 \mathrm{~min}$

$\Delta$ CRITICAL STEP The instrument should be calibrated daily to ensure high mass accuracy.

11. Fill the glass syringe ( $10 \mathrm{~mL}$ Fixed Luer Lock Tip Syringe, SGE, cat. no. 008970) with 0.5 $\mathrm{mM}$ sodium formate calibration solution.

12. Initiate MS scanning by pressing the 'On' ('I') button on the MassLynx ${ }^{\top M}$ tune page.

13. On the front panel of the solvent pump, set up the solvent flow rate at $0.2 \mathrm{~mL} / \mathrm{min}$ and initiate the flow by pressing the 'Run' button.

14. Wait until the sodium formate spectrum is visible and stable on the MassLynx ${ }^{\mathrm{TM}}$ tune page (multiple high peaks distributed across entire $\mathrm{m} / \mathrm{z}$-range).

15. Select the 'MS console' tab in the MassLynx ${ }^{\mathrm{TM}}$ tune page and subsequently the 'IntelliStart' tab. Set the system to 'Configuration mode' by choosing it in the 'Configure' drop-down menu at the top of the page.

16. Tick the empty box next to the 'Create Calibration' option on the instrument configuration panel and press 'Start'.

17. The step-by-step create calibration wizard algorithm will appear on the screen. Press 'Create Profile Editor...', then select 'File' drop-down from the pop-up window and press 'New' to define a new calibration profile.

18. Populate the 'New calibration profile window' by defining the profile name, setting the mass range from 50 to $1200 \mathrm{Da}$ and the calibration type as 'Assisted'. 
812 19. In the calibration profile window click 'Edit...' next to the 'Positive polarity' section. In the

813 new window choose 'Enabled' as an option for calibration reference compound and

814 select sodium formate in the drop-down menu.

815 20. Repeat step 15 for the 'Negative polarity' section within the calibration profile window.

816 - PAUSE POINT Once created, the calibration profile can be updated each time a new

817 instrument calibration is performed by resetting the already calibrated profile through

818 the 'Edit' drop-down menu in the Calibration profile editor. The reset profile will save all

819 defined calibration settings but lacks calibration data, so it can be used for new

820 calibration (the 'green tick' next to the calibration profile will change to 'orange triangle'

821 in the Calibration Profile pull down menu).

822 21. Choose both sensitivity and resolution mode for positive and negative polarity

823 calibration by ticking the corresponding boxes in the Calibration set-up wizard page.

824 22. Check both boxes ('Display Report' and 'Make Calibration Profile Active') in the 'Options' 825 section.

826 23. Do not change any settings on the other pages of the Create Calibration wizard sequence

827 and click the 'Next' button on the bottom of every page to reach the end of the 828 sequence.

829 24. Press the 'Start' button at the end of the Create Calibration wizard sequence. The $830 \quad$ calibration process will be initiated by the software.

831 25. At the end of the calibration process, the Calibration report for evaluation pops up. All

832 peaks in the acquired datafile should be matched with the reference file for sodium 833 formate (100\% peaks detected), the RMS residual mass and $95 \%$ confidence band should 834 be below 5 ppm mass deviation. 
835 26. If the abovementioned criteria for good system calibration are met, accept the 836 calibration report by clicking on the green 'tick' symbol above on the Calibration report

837 page. Continue the evaluation for both modes (sensitivity and resolution) and both 838 polarities (negative and positive).

839 27. When calibration has been successfully finalized, a new calibration file will be $840 \quad$ automatically saved and uploaded on the MS control panel.

841 28. Remove the remaining calibration solution from the syringe and replace the solvent to 842 isopropylalcohol, start the solvent pump and initiate the MS acquisition. Flush 843 approximately $20 \mathrm{~min}$ to remove the remaining calibration solution from the system.

844 29. After approximately $20 \mathrm{~min}$, check the tune page MS spectrum; no additional peaks aside 845 from the isopropylalcohol ions should be present within the spectrum TIC.

846

\section{Sample analysis}

848 30. Depending on whether a manual or automated sampling analysis will be performed, 849 follow the steps in options A or B.

850 A. Manual analysis $\bullet$ Timing $30 \mathrm{~s} /$ sample (per ionization mode)

851 (i) In the MassLynx ${ }^{\mathrm{TM}}$ software window, initiate MS acquisition by selecting all 852 samples in the sample sequence that should be analysed within one batch 853 and press the 'Run sequence' button on top of the page.

854 (ii) Activate the laser by pressing the 'Start' button next to the flash lamp and Q-

855 switch indicators on the laser control panel.

856

(iii) Place the microscope slide, carrying the sample, under the laser focus point for ablation; remove after $3 \mathrm{~s}$. 
(iv) (Optional) Repeat step (iii) to generate several replicates. If you do this, record 3 to $4 \mathrm{~s}$ (blank background signal) between burns for optimal peak resolution.

(v) Repeat steps (i) to (iv) for any other sample to be analysed.

$\Delta$ CRITICAL STEP Routinely clean the laser focusing lens from the side of ablation with a

863 cotton bud dipped in ethanol every 5 to 10 burns depending on the normal rate of

864 contamination. Clean the focusing lens in a similar fashion whenever the intensity of

865 acquired spectra decreases with more than $10 \%$.

866 (vi) (Optional) Repeat steps (i) to (v) for all samples when analysing the samples in

867 the opposite polarity mode, changing to the appropriate experimental

868 settings ('Equipment set-up’ section).

869 B. Automated analysis • Timing $23 \mathrm{~s} /$ sample (per ionization mode)

870 (i) Position the microtiter plate(s), containing the samples, on the sampling $871 \quad$ surface.

872 (ii) Ensure that the laser focus point lies in the middle of the first well (check with 873 the alignment diode beam, see Steps 1-8 for external optics alignment $874 \quad$ verification as described in Box 2).

875 (iii) Within the laser controlling software, the following experimental sequence 876 should be programmed: a 3-s burn followed be a 20.6-s cooldown period.

877 (iv) Within the stage controlling software, define the number of sample-filled 878 wells to be analysed (i.e. the number of repetitive movements that the stage 879 has to perform to position every sample-filled well under the laser focus 880 point). The moving stage 'move and wait' cycle timing should correspond to 881 the laser sequence program cycle of $23.6 \mathrm{~s})$. 
882

883

884

885

886

887

888

889

890

891

892

893

894

895

896

897

898

899

900

901

902

903

904

905

(v) Initiate the autosampler robot sequence.

(vi) After $5 \mathrm{~s}$ initiate the MS instrument acquisition sequence. This will set the burn 'retention time' at approximately $15 \mathrm{~s}$ in the acquisition file.

$\triangle$ CRITICAL STEP Clean the laser focusing lens from the side of ablation with a cotton bud dipped in ethanol every 10 to 15 burns.

(vii) (Optional) Repeat steps (iv)-(vii) for all samples when analysing the samples in the opposite polarity mode, changing to appropriate experimental settings ('Equipment set-up' section).

\section{Computational analysis • Timing $1 \mathrm{~h} / 500$ samples}

31. Load the acquired spectra into the appropriate software.

A CRITICAL STEP Data collected on the LA-REIMS instrument (Waters) are processed sequentially by Progenesis ${ }^{\circledR}$ Bridge (v1.0.29, Waters) and Progenesis ${ }^{\circledR}$ QI (V2.1, Waters); see Box 3 Steps 1-11.

32. Apply pre-processing to the spectra, including noise removal, baseline subtraction, selecting burns and peak picking.

33. Load the peak lists ( $\mathrm{m} / \mathrm{z}$-features) into the appropriate software.

$\triangle$ CRITICAL STEP The choice of software will depend on the classification modelling approach selected (commercial, open-source software) and compatibility with the output format after pre-processing. Here, data collected from Progenesis ${ }^{\circledR}$ QI (v2.1, Waters) are loaded into SIMCA (v15, Sartorius); see Box 3 Steps 12-22.

34. Select features that show statistical and significant differences in the discriminative analysis. 
35. After the experiment, and as part of troubleshooting, you will need to clean and maintain the intruments. Follow the guidelines in Table 1 for maintenance methods and frequency rate for each hardware component of the LA-REIMS set-up.

910

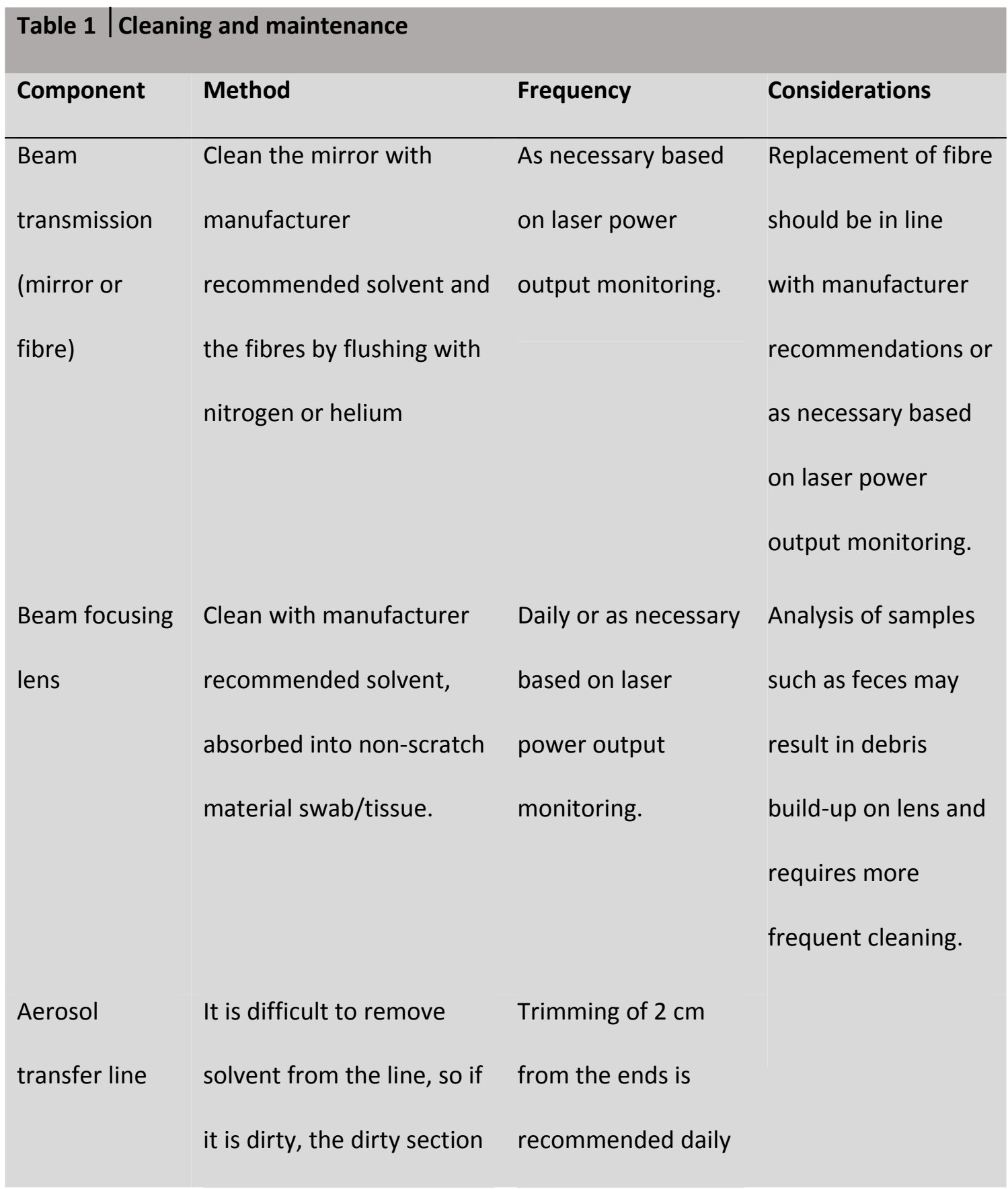




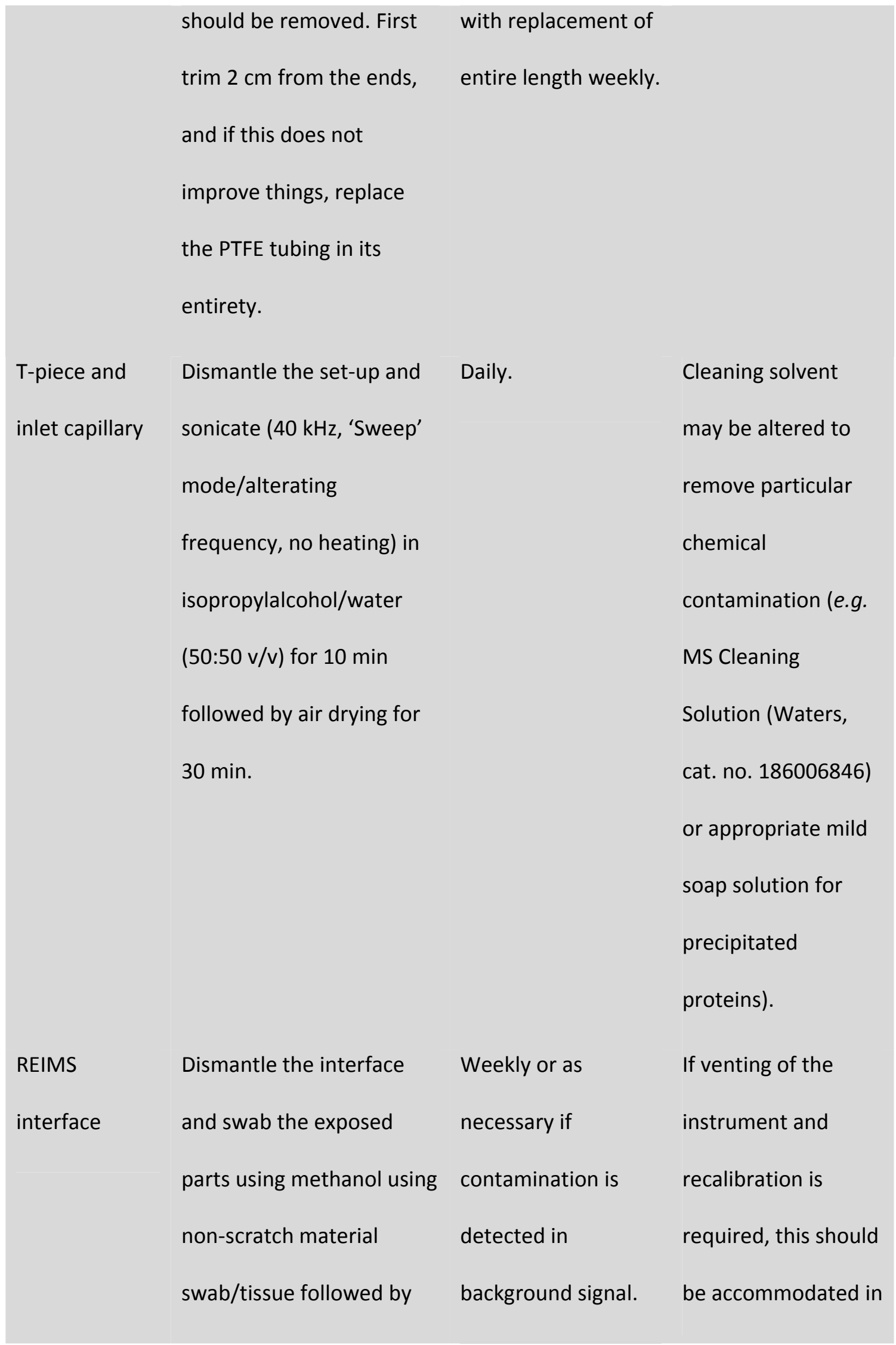




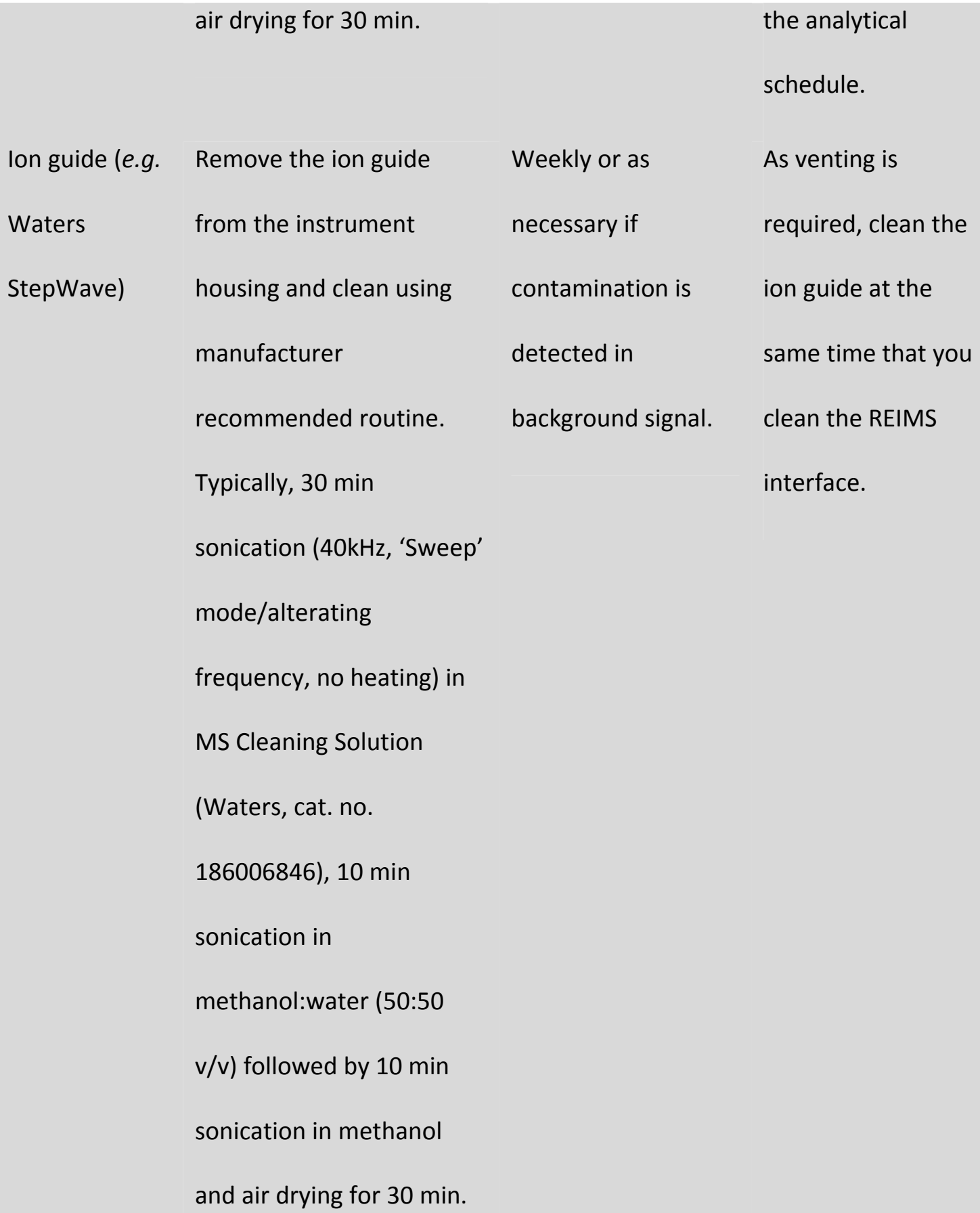


Table 2 |Troubleshooting table

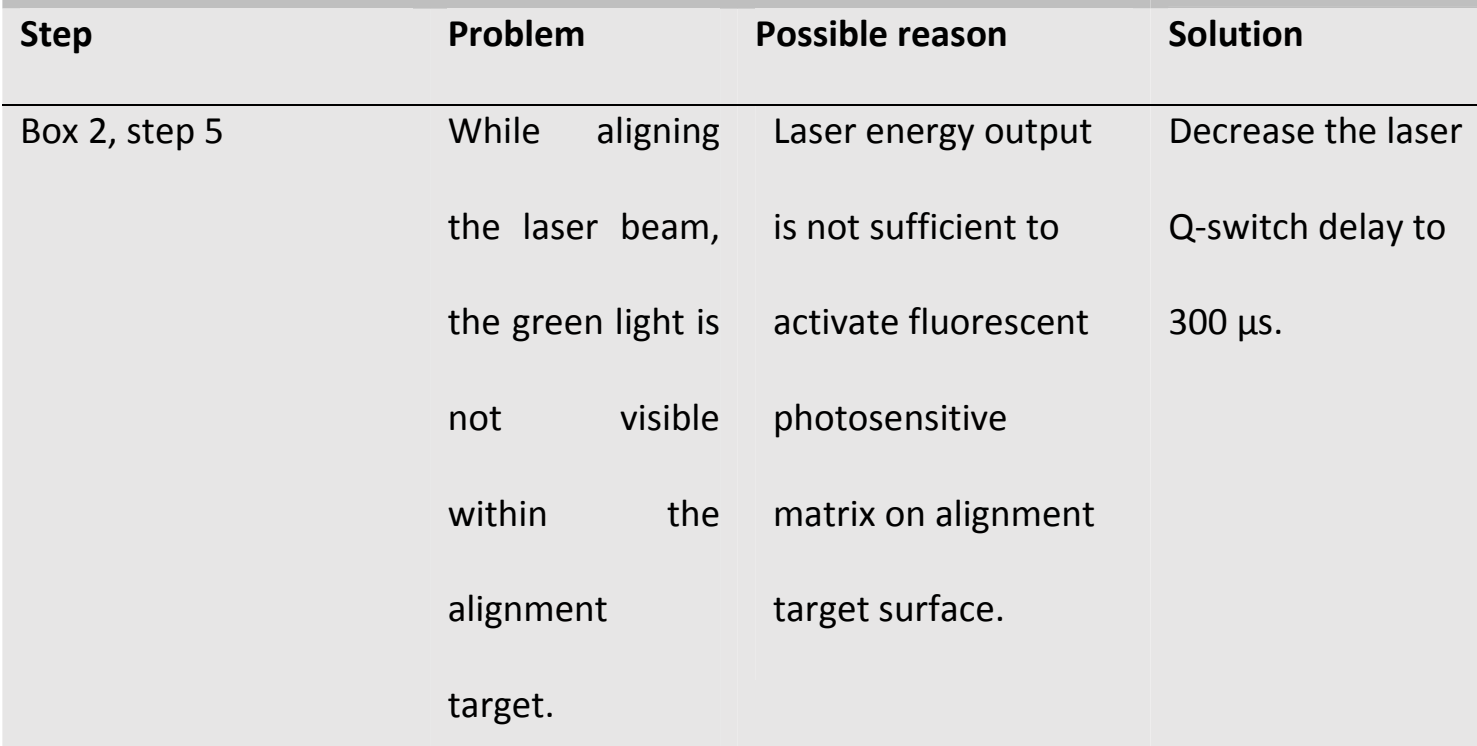

Box 3, step 1c

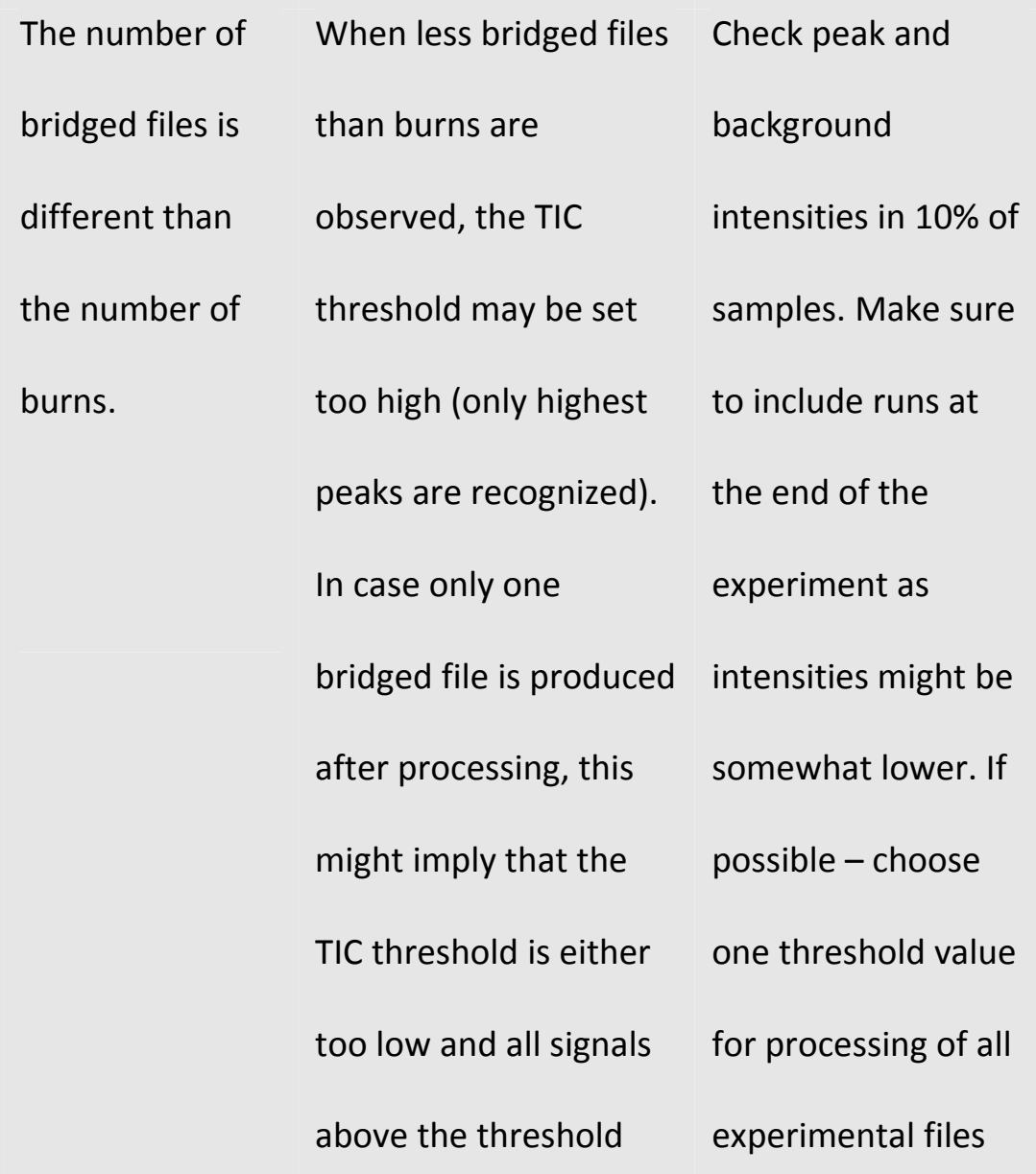




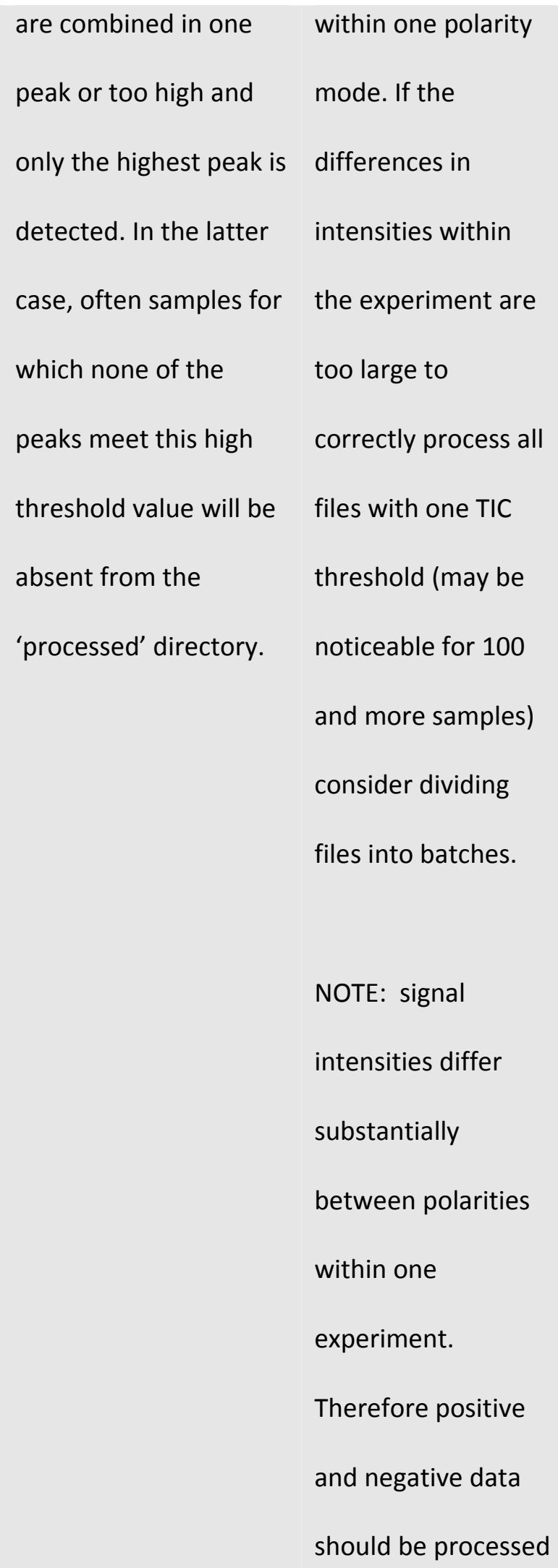


separately with

separate TIC

threshold values.

\begin{tabular}{|c|c|c|c|}
\hline Box 3, Step 15c & $\begin{array}{l}\text { The errors 'no } \\
\text { valid values', }\end{array}$ & $\begin{array}{l}\text { No values present in } \\
\text { the dataset. }\end{array}$ & $\begin{array}{l}\text { Check if all } \\
\text { '\#DIV/O!', 'NA', ' } \infty \text { ', }\end{array}$ \\
\hline & $\begin{array}{l}\text { 'missing } \\
\text { observation ID' } \\
\text { or 'missing } \\
\text { variable ID' pop } \\
\text { up. }\end{array}$ & 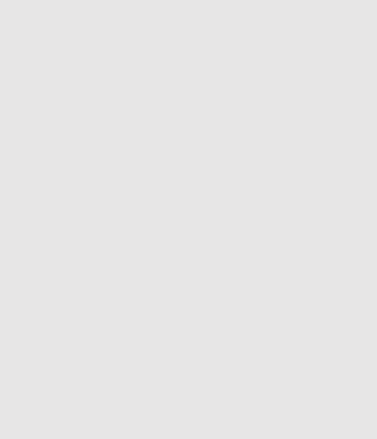 & $\begin{array}{l}\text {... values are } \\
\text { removed from the } \\
\text { dataset, including } \\
\text { the header } \\
\text { information. }\end{array}$ \\
\hline Step 8 & $\begin{array}{l}\text { While infusing } \\
\text { pure } \\
\text { isopropylalcohol, } \\
\text { the tune page } \\
\text { spectrum } \\
\text { displays } \\
\text { additional } \mathrm{m} / \mathrm{z} \\
\text { signals. }\end{array}$ & $\begin{array}{l}\text { Source, solvent line or } \\
\text { sample tube may be } \\
\text { contaminated. }\end{array}$ & $\begin{array}{l}\text { Perform additional } \\
\text { cleaning and/or } \\
\text { change infusion } \\
\text { solvent. }\end{array}$ \\
\hline
\end{tabular}

$916 \quad$ Timing

917 Step 1, Set-up of the sample holder or recipient: $1-2 \mathrm{~min} / \mathrm{sample}$ 
Step 2, Set-up of the laser: 10-15 min

919 Steps 3-6, Connecting REIMS sampling tube, solvent line and MS source: 5-10 min

920 Steps 7-10, System preparation and optimization: $20-25$ min

921 Steps 11-29, External calibration of the REIMS interface: 40 min

922 Step 30A, Manual analysis: 30 s/sample

923 Step 30B, Automated analysis: 23 s/sample

924 Steps 31-34, Computational analysis: $1 \mathrm{~h} / 500$ samples

925 Step 35, Cleaning and maintenance: $1.5 \mathrm{~h}$

926 Box 1, Sample preparation: 0-15 min/sample

927 Box 2, Laser alignment verification: 20-30 min

928 Box 3, LA-REIMS data analysis \& classification modelling: $1 \mathrm{~h} / 500$ samples

929

930 Anticipated results

931

With this protocol, we provide guidelines to perform rapid LA-REIMS metabolic

932 fingerprinting of various human biofluids, i.e. saliva, urine, plasma, stool, sputum, and milk.

933 Hereby, aspects on both sample handling and instrumental set-up are detailed. Using the

934 outlined procedures, high-quality data for a broad $\mathrm{m} / \mathrm{z}$-spectrum (50 - $1200 \mathrm{Da}$ ) can typically

935 be acquired in less than 1 min per sample; including sample loading and inter-sample

936 equilibration. No significant pre-treatments of the biofluids are needed, with the exception

937 of saliva. As such, LA-REIMS offers relevant potential for diverse clinical applications, in

938 which rapid discriminative metabotyping and/or (point-of-care) personalized health care

939 provision are major objectives. In this section, we discuss the anticipated results as may be

940 obtained upon biofluid LA-REIMS analysis, with specific elaborations on the generated

941 molecular fingerprints, the precision of the analysis, and the application potential in a clinical 
942 context. For this, we chose feces as a semi-fluid and saliva as a real biofluid, as examples for

943 in-depth illustration of some particular aspects.

944

945 Molecular fingerprints

946 For all biofluids and semi-fluids, the mass spectra that are generated by LA-REIMS comprise

947 several hundreds of features across the $50-1200$ Da $\mathrm{m} / \mathrm{z}$ scan range, for both negative and

948 positive ionization mode. In Fig. 5, we show representative molecular fingerprints for saliva,

949 plasma, urine, stool, milk, and sputum, as obtained from a single burn of a pooled sample (n $950=10)$.

951 We did a similar experiment where we analysed the samples by DIMS and have included the

952 methods and results for this in the Supplementary Discussion. From these results it was clear

953 that we were able to get a higher metabolome coverages using LA-REIMS. There was also

954 substantial contamination at the ionization source for DIMS making robust analysis of large

955 sample batches problematic. While these problems can be overcome using appropriate

956 sample pre-treatment for direct infusion MS of biofluids (e.g. saliva, urine, blood) and even

957 essential for semi-fluids (e.g. feces), this requirement detracts from the pursuit of rapid

958 (real-time) fingerprinting, as is achieved by LA-REIMS.

959 For the various matrices, the total number of unique features that could be retrieved by

960 considering the samples from ten individuals was 3628 for saliva $(52.0 \%$ of these were

961 detected in negative ionization mode), 3015 for plasma (47.2\% in negative), 3767 for urine

962 (54.1\% in negative), 2078 for feces (65.9\% in negative), 3065 for milk (31.6\% in negative),

963 and 2039 for sputum (35.1\% in negative).

964 In-depth analysis of the metabolic fingerprints shows that the lower $\mathrm{m} / \mathrm{z}$-region of $50-400$

965 Da generally contributes the most to the achieved metabolome coverage (number of 
detected features) for negative ionization mode, but the $\mathrm{m} / \mathrm{z}$ region of $200-600 \mathrm{Da}$

967 contributes the most for positive ionization mode (Fig. 6). This most likely relates to the

968 specific metabolome composition of the assessed matrices and the number of metabolite

969 representatives for the various chemical classes (i.e., reflected by various functional groups

970 and associated ionization behaviours). Also UHPLC-HRMS analysis revealed specific $\mathrm{m} / \mathrm{z}$ -

971 patterns, corresponding to the ionization mode, as e.g. illustrated for saliva by Wijnant et al.

$972(2020)^{13}$.

973

974 Taking into account the absolute metabolome coverage per matrix and making the 975 comparison between matrices, it may be noted that the metabolome of feces and milk 976 contain a relatively low proportion of high-weight features (> $800 \mathrm{Da})$, whereas the sputum 977 metabolome encompass a relatively low proportion of low-weight features $(<200 \mathrm{Da})$. It has 978 indeed been described that sputum especially contains a high fraction of various lipid 979 species, including highly abundant sphingolipids such as sphingomyelins, ceramides, and 980 lactosylceramides ${ }^{43}$. In human milk, a high abundance of various low- to medium-weight 981 metabolites have been reported, such as oligosaccharides, amino acids and derivatives, and 982 fatty acids and associated metabolites ${ }^{44}$. For feces, the defined metabolome composition 983 may largely be determined by the metabolic activity of the gut microbial community, 984 including the metablization of large and complex structures ${ }^{45}$.

985

986 The above-mentioned data were obtained for each biofluid based on a single burn. A typical 987 burn for positive and negative ionization is presented in Fig. 3. Taking into account the scan 988 time (see 'Equipment set-up' section), the total number of scans acquired per burn varied 989 between 4.2 scans (plasma) and 10.0 scans (feces). A higher background level was generally 
990 obtained in positive ionization mode $^{7}$ for all matrices, as displayed for feces (Fig. 3). It was

991 verified that especially $m / z$-features $<350$ Da contributed to this background signal.

992

\section{Precision}

994 As a major parameter to assess the performance quality, the precision of the LA-REIMS 995 method was verified for feces as a semi-fluid representative and saliva as a real biofluid. 996 Repeatability was evaluated based on the repetitive $(n=3)$ laser ablation of a single sample 997 as well as the sequential analysis of ten different samples from the same sample pool. 998 Reproducibility was assessed for three consecutive analysis days whereby ten sample 999 aliquots were analysed per day, prepared from the same sample pool, stored at $-80{ }^{\circ} \mathrm{C}$, and 1000 all subjected to one freeze-thaw cycle.

1001

1002 For the repeatability that was assessed by subjecting a single sample to three consecutive 1003 ablation events, it was observed that the achieved metabolome coverage was highly 1004 repeatable across the various burns and this for both ionization modes and matrices (Table 10053 , minimum of $84.5 \%$ congruence). With respect to the total ion current (TIC), it was noted 1006 that significant differences may occur from burn to burn (Table 3, CV\% up to $45.5 \%$ ). This is 1007 evidence that TIC-based data normalization is essential to attain high-quality LA-REIMS 1008 metabolomics datasets. Indeed, by applying TIC-based normalization, high signal 1009 repeatability was calculated with $\geq 92.5 \%$ of the features found in feces having a CV $\leq 30 \%{ }^{46}$; 1010 the corresponding value for saliva was $\geq 80.9 \%$. Based on the achieved metabolome 1011 coverages that were highly similar across burns and the high signal repeatability upon TIC1012 based normalization, acquisition of representative LA-REIMS metabolic fingerprints is 1013 possible based on one burn only. 


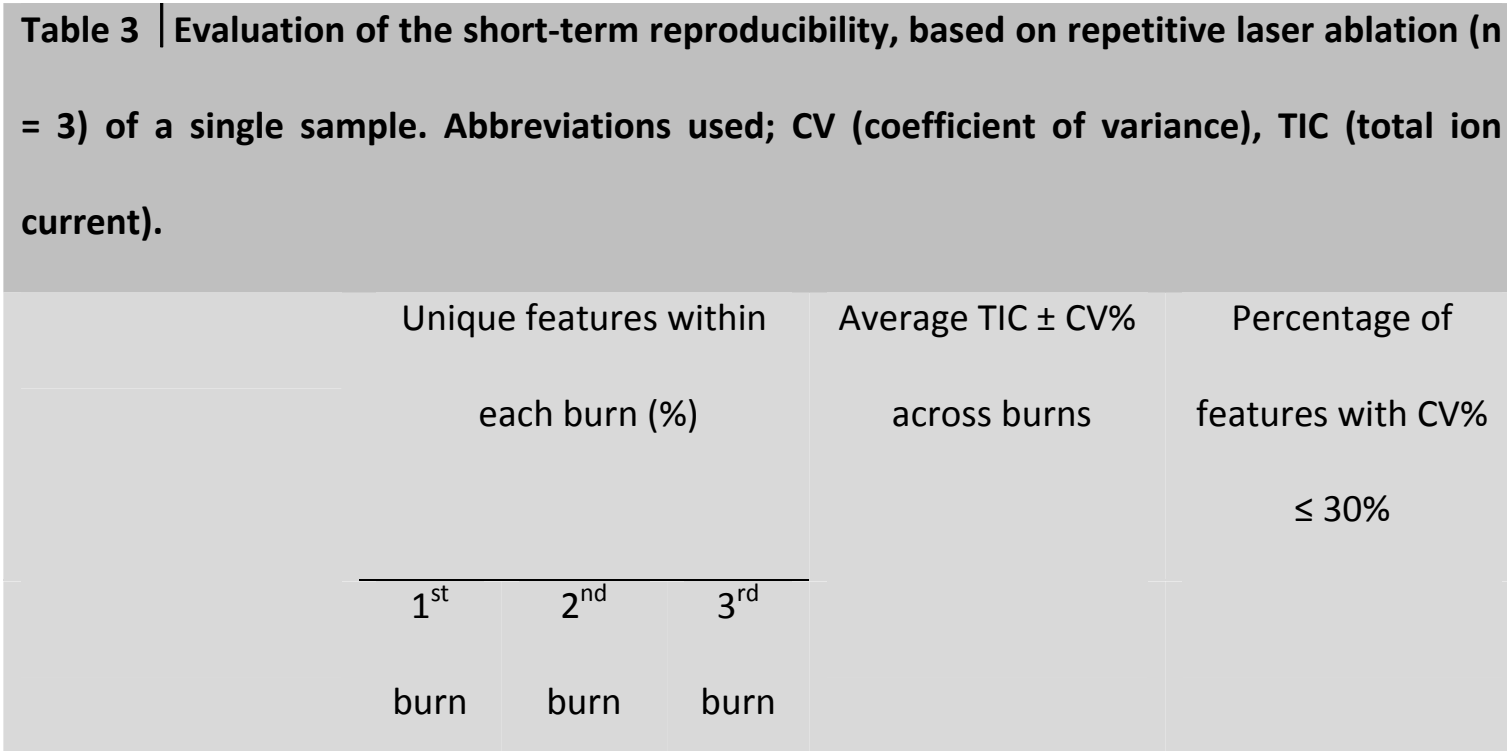

\section{Feces}

$\begin{array}{llllll}\text { Positive ionization } & 9.4 & 4.1 & 6.1 & 7.8 \mathrm{e}^{7} \pm 28.1 & 92.5\end{array}$
Negative
3.7
0.4
4.3
$3.8 e^{8} \pm 45.4$
96.4

ionization

Merged polarities $\quad 5.4 \quad 1.6 \quad 4.8$

95.5

\section{Saliva}

\begin{tabular}{|c|c|c|c|c|c|}
\hline Positive ionization & 2.6 & 1.7 & 1.5 & $3.6 e^{8} \pm 7.7$ & 83.4 \\
\hline $\begin{array}{l}\text { Negative } \\
\text { onization }\end{array}$ & 0.5 & 0.2 & 1.3 & $1.9 \mathrm{e}^{8} \pm 45.5$ & 80.9 \\
\hline Merged polarities & 1.5 & 0.9 & 1.4 & & 82.0 \\
\hline
\end{tabular}

1016 Evaluation of the intra-day repeatability for the ten consecutive sample analyses was

1017 achieved by assessing the similarity of the molecular profiles in terms of metabolome

1018 coverage and by calculating the CV\% for the TIC-normalized feature abundances. For feces 
and saliva, metabolome coverage was considered highly repeatable in terms of number of

1020 features detected (Table 4). Moreover, the CV\% as determined for the TIC-normalized

1021 features' abundances indicated that $71.8 \%$ of the fecal features and $88.9 \%$ of the salivary

1022 features meet the $30 \%$ CV-threshold, typically used in metabolomics analysis ${ }^{46}$. Inter-day

1023 repeatability (i.e. the reproducibility) results were generally quite similar to those obtained

1024 for the intra-day repeatability assessment, indicating that high-quality data acquisition by

1025 LA-REIMS can be actualized for both biofluid and semi-fluid analysis over the course of

1026 several days (Table 4). Hereby, internal QC-samples can be used as an efficient strategy to

1027 adjust for possible instrumental variation (LOESS-based normalization).

1028 It should be noted, however, that for feces in positive ionization mode, only $38.9 \%$ of the

1029 detected features had an acceptable $\mathrm{CV} \leq 30 \%$. This may partially relate to the fact that a

1030 higher noise level is observed in positive ionization mode, which may somewhat hamper

1031 reproducible analysis.In addition, microbial enzymatic activity may reactivate after preparing

1032 the fecal slurry, as a result of which the metabolic composition of the pooled sample may be

\section{3 altered.}

1034

Table 4 Evaluation of the short-term repeatability (10 samples, intra-day) and longterm reproducibility (3 days, every day 10 samples). Abbreviations used; CV (coefficient of variance).

\begin{tabular}{|c|c|c|c|}
\hline \multicolumn{2}{|c|}{ Short-term reneatability } & \multicolumn{2}{|c|}{ Long-term reproducibilitv } \\
\hline \multicolumn{2}{|c|}{ (10 samples) } & \multicolumn{2}{|c|}{ ( 3 days, 10 samples per day } \\
\hline Average & Percentage & Average & Percentage \\
\hline number of & features with CV & number o & features \\
\hline
\end{tabular}




\begin{tabular}{|c|c|c|c|c|}
\hline & features $\pm \mathrm{CV} \%$ & $\leq 30 \%$ & features $\pm \mathrm{CV} \%$ & $C V \leq 30 \%$ \\
\hline \multicolumn{5}{|l|}{ Feces } \\
\hline $\begin{array}{l}\text { Positive } \\
\text { ionization }\end{array}$ & $546 \pm 3.8$ & 67.9 & $528 \pm 6.5$ & 38.9 \\
\hline Negative & $1217 \pm 3.0$ & 73.5 & $1193 \pm 3.8$ & 70.3 \\
\hline \multicolumn{5}{|l|}{ ionization } \\
\hline $\begin{array}{l}\text { Merged } \\
\text { polarities }\end{array}$ & $1765 \pm 2.2$ & 71.8 & $1721 \pm 3.4$ & 66.6 \\
\hline \multicolumn{5}{|l|}{ Saliva } \\
\hline $\begin{array}{l}\text { Positive } \\
\text { ionization }\end{array}$ & $1079 \pm 3.2$ & 82.6 & $1090 \pm 2.4$ & 78.8 \\
\hline $\begin{array}{l}\text { Negative } \\
\text { ionization }\end{array}$ & $1278 \pm 0.5$ & 94.3 & $1178 \pm 0.4$ & 88.7 \\
\hline Merged & $2357 \pm 1.4$ & 88.9 & $2284 \pm 2.3$ & 82.6 \\
\hline polarities & & & & \\
\hline
\end{tabular}

\section{Classification model}

1037 To demonstrate the application potential of LA-REIMS for rapid fingerprinting of bio/semi1038 fluids and to substantiate the robustness of the methodology, this section describes two 1039 example clinical cases, i.e. type 2 diabetes and overweight/obesity. For these cases, LA1040 REIMS discriminative fingerprinting according to health status was pursued by considering, 1041 respectively, a semi-fluid (feces) and real biofluid (saliva). 
1043 Based on the fecal LA-REIMS fingerprints, a valid two-class OPLS-DA model was established

1044 to discriminate between individuals with type 2 diabetes and those with normal glycaemic

1045 state (i.e. euglycemia). Also, the PCA-X score plot (Fig. 7) showed some segregation of 1046 samples according to health status. The OPLS-DA model (Fig. 7) was validated with a CV-

1047 ANOVA $p$-value of $1.93 \mathrm{e}^{-17}$, valid permutation testing, and $R^{2}(X)$ of $0.230, R^{2}(Y)$ of 0.946 and

$1048 \mathrm{Q}^{2}(\mathrm{Y})$ of 0.734 . Moreover, using a five-fold $20 \%$-leave out validation strategy, a general

1049 classification accuracy of $90.5 \%$ was calculated, with a type 2 diabetes sensitivity of $89.5 \%$

1050 and specificity of $91.7 \%$. Initial classification of individuals according to glycaemic state was

1051 based on the glycated haemoglobin concentration (HbA1c), thereby implementing a 60

$1052 \mathrm{mmol} / \mathrm{mol}$ threshold criterion. A total of 72 individuals took part in the study, whereby 36

1053 individuals had been assigned a state of type 2 diabetes and 36 individuals a state of

1054 euglycemia. A detailed explanation on the sample collection, data analysis, data processing

1055 and interpretation of the results can be consulted in Van Meulebroek et al. (2020) ${ }^{12}$.

1056 Whereas the main purpose of this experiment was the rapid discrimination and thus

1057 diagnosis of glycaemic state based on rapid LA-REIMS metabolic fingerprinting of the semi-

1058 fluid feces, the instrumental platform also allows for some more in-depth interpretation of

1059 discriminative metabolites (i.e. potential markers). To this end, samples can be subjected to

1060 MS/MS fragmentation, which offers possibilities for tentative identification. In our study,

1061 this fragmentation strategy allowed to assign relevant metabolite identities, substantiating

1062 the method also suited for exploration of the mechanisms involved in pathophysiology.

1063 Amongst others, 6-hydroxymelatonin, several nicotinamides, D-glucose, biotripyrrin, and

1064 homocysteine-associated metabolites were tentatively identified as metabolite markers. As

1065 such, applying LA-REIMS fingerprinting, classification according to glycaemic state was 
1066 considered possible based on validated supervised modelling, biological qualification of

1067 tentatively identified metabolite markers, and supportive data from UHPLC-HRMS analysis ${ }^{12}$.

1068

1069 To assess overweight and obesity in young adolescents (6 to 16 years), saliva samples from

107035 individuals with a healthy weight and 35 individuals with overweight/obesity were

1071 collected and subjected to LA-REIMS analysis. Hereby, the primary purpose was to achieve

1072 discrimination according to weight status, as this may enclose interesting opportunities to

1073 appoint metabolic perturbations according to pathophysiological state and evaluate e.g. the

1074 contribution of etiological factors and treatment efficacy. Initial adiposity classifications

1075 were based on BMI z-scores (adjusted BMI for age and sex), following Roelants et al. (2009) ${ }^{47}$

1076 and extended international (IOTF) body mass index cut-offs for thinness, overweight, and

1077 obesity by Cole et al. (2012) ${ }^{48}$. A valid two-class OPLS-DA model (Fig. 7) could be established,

1078 which allowed for discrimination between healthy weight (IOTF of 0$)$ and

1079 overweight/obesity (IOTF $\geq 1$ ). Validation parameters comprised a CV-ANOVA $p$-value of

$10809.01 \mathrm{e}^{-21}$, valid permutation testing, and an $\mathrm{R}^{2}(\mathrm{X})$ of $0.227, \mathrm{R}^{2}(\mathrm{Y})$ of 0.946 , and $\mathrm{Q}^{2}$ of 0.808 . In

1081 addition, based on a five-fold 20\%-leave out validation strategy, a general classification

1082 accuracy of $97.1 \%$ was achieved, with a sensitivity of $100 \%$ and specificity of $94.3 \%$. A

1083 detailed explanation on the sample collection, data analysis, data processing and 1084 interpretation of the results can be consulted in Wijnant et al. $(2020)^{13}$.

1085

1086

1087 Reporting summary: Further information on research design is available in Nature Research 1088 Reporting Summary linked to this article.

1089 
1091 Datasets relevant to our published supporting primary papers can be made available from

1092 the corresponding author upon reasonable request. The source data for figures is publicly 1093 available at Figshare repository:

1094 Fig. 3 https://doi.org/10.6084/m9.figshare.14258423.v1;

1095 Fig. 5 https://doi.org/10.6084/m9.figshare.14258438.v1;

1096 Fig. 6 https://doi.org/10.6084/m9.figshare.14258459.v2

1097

1098

References:

1099

1100 1. Gong, Z., Zhang, J. \& Xu, Y.-J. Metabolomics Reveals that Momordica charantia

1101 Attenuates Metabolic Changes in Experimental Obesity. Phyther. Res. 31, 296-302

$1102 \quad$ (2017).

1103 2. Pham-Tuan, H., Kaskavelis, L., Daykin, C. A. \& Janssen, H. G. Method development in

1104 high-performance liquid chromatography for high-throughput profiling and

1105 metabonomic studies of biofluid samples. J. Chromatogr. B Anal. Technol. Biomed. Life

1106 Sci. 789, 283-301 (2003).

1107 3. Wishart, D. S. Metabolomics for investigating physiological and pathophysiological

$1108 \quad$ processes. Physiol. Rev. 99, 1819-1875 (2019).

1109 4. Wijnant, K. et al. Validated Ultra-High-Performance Liquid Chromatography Hybrid $1110 \quad$ High-Resolution Mass Spectrometry and Laser-Assisted Rapid Evaporative Ionization

1111 Mass Spectrometry for Salivary Metabolomics. Anal. Chem. 92, 5116-5124 (2020).

1112 5. Johnson, C. H., Ivanisevic, J. \& Siuzdak, G. Metabolomics: Beyond biomarkers and 1113 towards mechanisms. Nat. Rev. Mol. Cell Biol. 17, 451-459 (2016). 
1114 6. Ferreira, C. R. et al. Ambient ionization mass spectrometry for point-of-care

1115 diagnostics and other clinical measurements. Clin Chem. 62, 99-110 (2016).

1116 7. Cameron, S. J. S. et al. Evaluation of Direct from Sample Metabolomics of Human

1117 Feces Using Rapid Evaporative Ionization Mass Spectrometry. Anal. Chem. 91, 13448-

$1118 \quad 13457$ (2019).

1119 8. Ogrinc, N. et al. Water-assisted laser desorption/ionization mass spectrometry for

1120 minimally invasive in vivo and real-time surface analysis using SpiderMass. Nat.

$1121 \quad$ Protoc. 14, 3162-3182 (2019).

1122 9. Schäfer, K. C. et al. In vivo, in situ tissue analysis using rapid evaporative ionization

1123 mass spectrometry. Angew. Chemie - Int. Ed. 48, 8240-8242 (2009).

1124 10. Clendinen, C. S., Monge, M. E. \& Fernández, F. M. Ambient mass spectrometry in 1125 metabolomics. Analyst 142, 3101-3117 (2017).

1126 11. Li, L., Hsieh, H. \& Hsu, C. Clinical Application of Ambient lonization. Mass spectrometry 1127 (Tokyo) 6, Spec Iss S0060 (2017).

1128 12. Van Meulebroek, L. et al. Rapid LA-REIMS and comprehensive UHPLC-HRMS for 1129 metabolic phenotyping of feces. Talanta 217, 121043 (2020).

1130 13. Wijnant, K. et al. Validated Ultra-High-Performance Liquid Chromatography Hybrid 1131 High- Resolution Mass Spectrometry and Laser-Assisted Rapid Evaporative Ionization 1132 Mass Spectrometry for Salivary Metabolomics. Anal. Chem. 92, 5116-5124 (2020).

1133 14. Fatou, B. et al. In vivo Real-Time Mass Spectrometry for Guided Surgery Application. 1134 Sci. Rep. 6, 1-14 (2016).

1135 15. Kuo, T. H., Dutkiewicz, E. P., Pei, J. \& Hsu, C. C. Ambient lonization Mass Spectrometry 1136 Today and Tomorrow: Embracing Challenges and Opportunities. Anal. Chem. 92, $1137 \quad 2353-2363(2020)$. 
1138 16. Sächfer, K.-C. et al. In situ, real-time identification of biological tissues by ultraviolet

1139 and infrared laser desorption ionization mass spectrometry. and infrared laser

1140 desorption ionization mass spectrometry. Anal. Chem. 83, 1632-1640 (2011).

1141 17. Balog, J. et al. Intraoperative Tissue Identificaition Using Rapid Evaporative Ionization

1142 Mass Spectrometry. Sci. Transl. Med. 5, 1-11 (2013).

1143 18. Feider, C. L., Krieger, A., Dehoog, R. J. \& Eberlin, L. S. Ambient Ionization Mass

1144 Spectrometry: Recent Developments and Applications. Anal Chem. 91, 4266-4290

1145 (2019).

1146 19. Fillet, M. \& Fre, M. Metabolomics in Medicinal Chemistry The emergence of

1147 metabolomics as a key discipline in the drug discovery process. Drug Discovery Today

$1148 \quad$ Technol. 13, 19-24 (2015).

1149 20. Golf, O. et al. Rapid evaporative ionization mass spectrometry imaging platform for

1150 direct mapping from bulk tissue and bacterial growth media. Anal. Chem. 87, 2527-

$2534(2015)$.

1152 21. Clendinen, C. S., Monge, M. E. \& Fernández, F. M. Ambient mass spectrometry in

1153 metabolomics. Analyst 142, 3101-3117 (2017).

$115422 . \quad$ Nemes, P. \& Vertes, A. Ambient mass spectrometry for in vivo local analysis and in situ

1155 molecular tissue imaging. TrAC - Trends Anal. Chem. 34, 22-34 (2012).

1156 23. Li, H., Balan, P. \& Vertes, A. Molecular Imaging of Growth, Metabolism , and

1157 Antibiotic Inhibition in Bacterial Colonies by Laser Ablation Electrospray lonization

1158 Mass Spectrometry. Angew Chem Int Ed Engl. 55, 15035-15039 (2016).

1159 24. Suva, M. A Brief Review on Dried Blood Spots Applications in Drug Development. J.

$1160 \quad$ Pharm. Biosci. 2, 17-23 (2014).

1161 25. Van Meulebroek, L. et al. Holistic Lipidomics of the Human Gut Phenotype Using 
Validated Ultra-High-Performance Liquid Chromatography Coupled to Hybrid Orbitrap

1163 Mass Spectrometry. Anal. Chem. 22, 12502-12510 (2017).

1164 26. De Paepe, E. et al. A validated multi-matrix platform for metabolomic fingerprinting of

1165 human urine, feces and plasma using ultra-high performance liquid-chromatography

1166 coupled to hybrid orbitrap high-resolution mass spectrometry. Anal. Chim. Acta 1033,

$1167 \quad 108-118(2018)$.

1168 27. Cameron, S. J. S. \& Takáts, Z. Mass spectrometry approaches to metabolic profiling of

1169 microbial communities within the human gastrointestinal tract. Methods (San Diego,

$1170 \quad$ Calif.) 149, 13-24 (2018).

1171 28. Gowers, G. O. F. et al. Off-Colony Screening of Biosynthetic Libraries by Rapid Laser-

1172 Enabled Mass Spectrometry. ACS synthetic biology 8, 2566-2575 (2019).

1173 29. Williams, J. P. \& Scrivens, J. H. Coupling desorption electrospray ionisation and neutral

1174 desorption/extractive electrospray ionisation with a travelling-wave based ion

1175 mobility mass spectrometer for the analysis of drugs. Rapid Commun. Mass Spectrom.

$1176 \quad 22,187-196(2008)$.

1177 30. Jones, E. A. et al. Matrix assisted rapid evaporative ionization mass spectrometry.

$1178 \quad$ Anal. Chem. 91, 9784-9791 (2019).

1179 31. De Spiegeleer, M. et al. Impact of storage conditions on the human stool metabolome $1180 \quad$ and lipidome: Preserving the most accurate fingerprint. Anal. Chim. Acta 1108, 79-88 $1181 \quad$ (2020).

1182 32. Laparre, J. et al. Impact of storage conditions on the urinary metabolomics fingerprint. $1183 \quad$ Anal. Chim. Acta 951, 99-107 (2017).

1184 33. Cheng, K., Brunius, C., Fristedt, R. \& Landberg, R. An LC-QToF MS based method for 1185 untargeted metabolomics of human fecal samples. Metabolomics 16, 46 (2020). 
1186 34. Zheng, J., Dixon, R.A . \& Li, L. Development of isotope labeling LC-MS for human

1187 salivary metabolomics and application to profiling metabolome changes associated

1188 with mild cognitive impairment. Anal. Chem. 84, 10802-10811 (2012).

1189 35. Pinto, J. et al. Human plasma stability during handling and storage: impact on NMR

$1190 \quad$ metabolomics. Analyst 139, 1168-1177 (2014).

$119136 . \quad$ Laparre, J. et al. Impact of storage conditions on the urinary metabolomics fingerprint.

$1192 \quad$ Anal. Chim. Acta 951, 99-107 (2017).

1193 37. Berkow, S. E. et al. Lipases and lipids in human milk: effect of freeze-thawing and

1194 storage. Pediatr. Res. 18, 1257-1262 (1984).

1195 38. Zhao, J., Evans, C. R., Carmody, L. A. \& LiPuma, J. J. Impact of storage conditions on

1196 metabolite profiles of sputum samples from persons with cystic fibriosis. J. Cyst.

$1197 \quad$ Fibros. 14, 468-473 (2015).

1198 39. Smith, C. A., Want, E. J., O’Maille, G., Abagyan, R. \& Siuzdak, G. XCMS: Processing

1199 mass spectrometry data for metabolite profiling using nonlinear peak alignment,

1200 matching, and identification. Anal. Chem. 78, 779-787 (2006).

1201 40. Pluskal, T. T., Castillo, S., Villar-Briones, A., Oresic, M. \& Orešič, M. MZmine 2: modular

1202 framework for processing, visualizing, and analyzing mass spectrometry-based

1203 molecular profile data. BMC Bioinformatics 11, 395 (2010).

1204 41. Abdi, H. Partial least squares regression and projection on latent structure regression.

1205 Wiley Interdiscip. Rev. Comput. Stat. 2, 97-106 (2010).

1206 42. Bylesjö, M. et al. OPLS discriminant analysis: combining the strengths of PLS-DA and

1207 SIMCA classification. J. Chemom. 20, 341-351 (2006).

1208 43. Quinn, R. A. et al. Microbial, host and xenobiotic diversity in the cystic fibriosis sputum

1209 metabolome. ISME J. 10, 1483-1498 (2016). 
1210 44. Smilowitz, J.T. et al. The human milk metabolome reveals diverse oligosaccharide

1211 profiles. J. Nutr. 143, 1709-1718 (2013).

1212 45. Matysik, S., Le Roy, C. I., Liebisch, G. \& Claus, S. P. Metabolomics of fecal samples: a 1213 practical consideration. Trends Food Sci. Technol. 57, 244-255 (2016).

1214 46. Naz, S., Vallejo, M., García, A. \& Barbas, C. Method validation strategies involved in 1215 non-targeted metabolomics. J. Chromatogr. A 1353, 99-105 (2014).

1216 47. Roelants, M., Hauspie, R. \& Hoppenbrouwers, K. References for growth and pubertal 1217 development from birth to 21 years in Flanders, Belgium. Ann. Hum. Biol. 36, 680-94 1218 (2009).

1219 48. Cole, T. J. \& Lobstein, T. Extended international (IOTF) body mass index cut-offs for 1220 thinness, overweight and obesity. Pediatr. Obes. 7, 284-294 (2012).

1221

1222

\section{Acknowledgements}

1224 This work was supported by VLAIO (grant number IM 150740 - A16/OC/0006), BOF GOA 1225 (grant number 2017/000102), BOF (grant number 01J07519), FWO Hercules (grant number 1226 AUG/17/09), FWO (grant numbers 1S57920N and 1S49020N) and ERC FWO Runner-up 1227 (grant number G0G0119N). The Lab of Chemical Analysis research group is part of the Ghent 1228 University expertise centre MSsmall.

1229

1230 Authors contributions

1231 V.P., L.V.M., M.D.G., M.D.S., E.D.P. and S.C. wrote the original draft of the manuscript. V.P., 1232 L.V.M., E.V.W. and A.P. carried out the experiments. M.D.G. and A.P. performed the data 1233 analysis. L.V.M., E.D.P. and S.C. collected the samples. Z.T. and S.C. developed the technique. 
1234 L.V., Z.T. and S.C. supervised the project and provided the funding. L.V., M.D.S. and L.V.M.

1235 performed the proofreading and correction of the manuscript.

1236

1237 Competing interests

1238 The authors declare that they have no known competing financial interests or personal

1239 relationships that could have appeared to influence the work reported in this paper.

\section{Additional information}

1241 Supplementary information is available for this paper at $x x x$.

1242 Reprints and permissions information is available at www.nature.com/reprints.

1243 Correspondence and requests for materials should be addressed to L.V. or S.C.

\section{Related links}

Key references using this protocol

Cameron, S. et al. Anal. Chem. 91 (21), 13488 (2019):

https://pubs.acs.org/doi/abs/10.1021/acs.analchem.9b02358

Wijnant, K. et al. Anal. Chem. 92 (7), 5116 (2020):

https://pubs.acs.org/doi/10.1021/acs.analchem.9b05598

Van Meulebroek, L. et al. Talanta 217, 121043 (2020):

https://www.sciencedirect.com/science/article/pii/S0039914020303349

FIGURES

1248 Fig. 1. A schematic representation of the LA-REIMS workflow. Technical scheme of the

1249 LA-REIMS set-up whereby a laser beam is focused onto a sample, being placed on either an 
1250 automated stage equipped to handle 96-well plates or a microscope slide for single samples.

1251 Rapid heating and evaporation takes place and the resulting analyte-containing aerosol is

1252 aspirated towards the mass spectrometer under the instrument's internal vacuum. The

1253 vapour is mixed with a solvent matrix containing lock mass / external standards and collides

1254 with a heated collision surface to form gas-phase ions for analysis. Ions are analysed using a

1255 quadrupole time-of-flight mass spectrometer and the resulting mass spectra are used in

1256 multivariate and machine learning classification models.

1257 - Okay. From this figure, I can see why the standards are referred to as "external".

1258 - Why are there two arrows for aerosol transfer?

1259 - I don't understand the graphic for the flight chamber.

1260 - To what extent is the multivariate modelling and machine learning specific to this

1261 type of experiment i.e. specific to LA-REIMS?

1262

1263

1264 Fig. 2. A flowchart of a standard LA-REIMS biofluid analysis experiment. Summary of

1265 steps involved in the implementation of LA-REIMS for molecular fingerprinting of biofluids,

1266 with time indications per step.

1267 - This is great

$1268 \quad-\quad$ Consider mentioning the other boxes in the flow diagram?

1269 - Move the mention of Box 1 to the blue box on the right?

1270

1271 Fig. 3. | Typical burns for LA-REIMS analysis. LA-REIMS burns as acquired for a stool sample

1272 in negative (a) and positive (b) ionization mode, with an MS scan time of $0.3 \mathrm{~s}$ per scan and

1273 (automated) laser ablation time of 3 s.x 
1274 From this it seems that the instrument is continuously scanning. I am intrigued about the

1275 baseline. I assume that this should be a constant that is made up of the solution that may or

1276 may not contain external standards.

1277

1278 Fig. 4.| Close-up of the motorized automated sampling area and its connection to the

1279 REIMS source: (a) Configuration of the motorised stage, with the laser beam focused on the

1280 surface of the stage and the sample tube positioned above the laser focus point. (b) Picture

1281 of the connection of the sample tube through the stainless-steel T-shaped connector piece

1282 with the solvent line and the inlet of the REIMS source.

1283

1284

1285

1286

1287

1288 instrument. Adaptation from Van Meulebroek et al. (2020) ${ }^{10}$ and Wijnant et al. (2020) ${ }^{11}$.

1289 - This is good as a quick "difference between sample types".

1290 - I wonder whether it would be good to have an annotated version showing some

1294 Fig. 6. | Typical $m / z$ profiles as obtained by LA-REIMS analysis of human biofluids. Number

of features that were measured per $\mathrm{m} / \mathrm{z}$-class upon LA-REIMS analysis of saliva, plasma,

1296 urine, feces, milk, and sputum, in positive (a) and negative (b) ionization mode. For each 
matrix type, a single list of $\mathrm{m} / \mathrm{z}$-features was acquired, based on the processing of ten rawfiles (single burns), as obtained from ten different samples (i.e. individuals). for each sample type might be more informative. i.e. the items on the $\mathrm{x}$ axis are the sample types, and the different coloured bars are different $\mathrm{m} / \mathrm{z}$ classes. The problem with this current figure is that it is difficult to see that sputum bas a different profile

Fig. 7. | Multivariate modelling of pathophysiological state based on LA-REIMS

fingerprints. PCA-X (a) and OPLS-DA (b) score plot upon LA-REIMS fingerprinting of feces to discriminate adults according to glycaemic state, i.e. type 2 diabetes (dark grey) (n = 36) and euglycemia (light grey) ( $\mathrm{n}=36)$. PCA-X (c) and OPLS-DA (d) score plot upon LAREIMS fingerprinting of saliva to discriminate adolescents according to weight status, i.e.

1310 healthy weight (dark grey) $(\mathrm{n}=35)$ and overweight/obesity (light grey) $(\mathrm{n}=35)$. Adaptation

1311 from Van Meulebroek et al. (2020) ${ }^{10}$ and Wijnant et al. $(2020)^{11}$. 


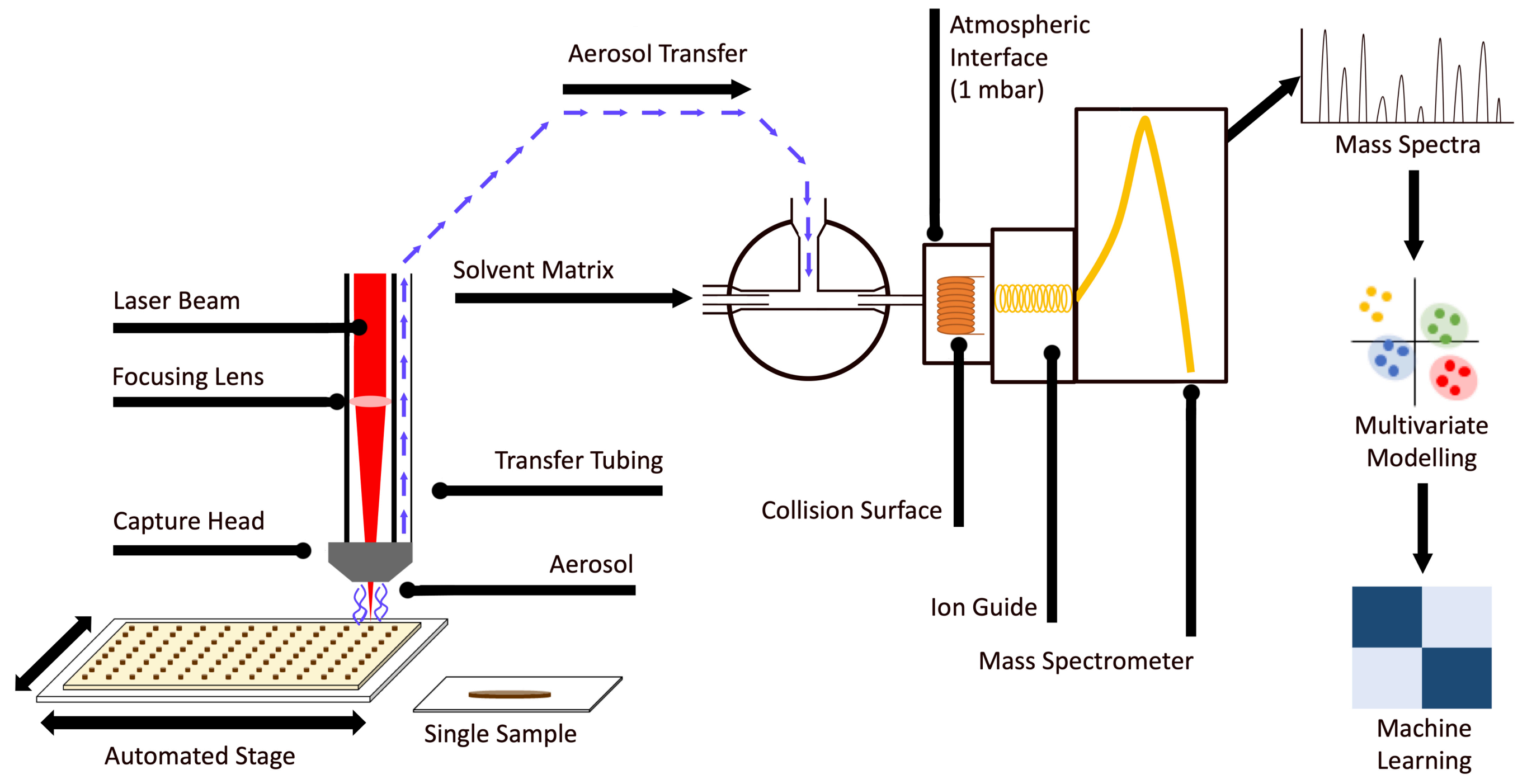


Sample collection

\section{Sample}

preparation needed?

Manually

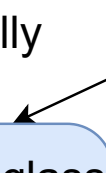

Transfer sample to glass microscope slide

(Step 1A or B)

\section{1-2 min/sample}

Automated

\section{performed manually}

or automated?

according to the matrixspecific protocol

(Box 1)

5-15 min/sample

\section{Analysis}

$1-2$ min/sample

Sampling tube and solvent line setup

(Steps 3-6)

(Step 2, Box 2)

10-15 min

5-10 min

MS instrument preparation and optimisation

(Steps 7-29)

20-25 min

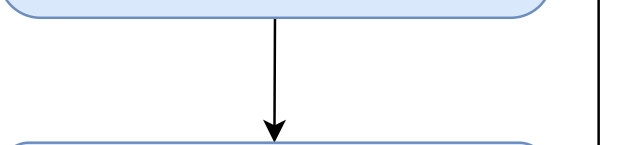

Sample analysis

(Step 30A or 30B)

20-30 s/sample

Data collection and computational analysis

(Steps 31-34, Box 3)
Transfer sample to microtiter plate

(Step 1C or 1D)
Follow cleaning and maintenance procedures to ensure stable performance

Consult the troubleshooting section when problems occur during system setup, sample or data analysis 

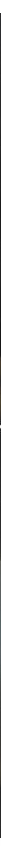

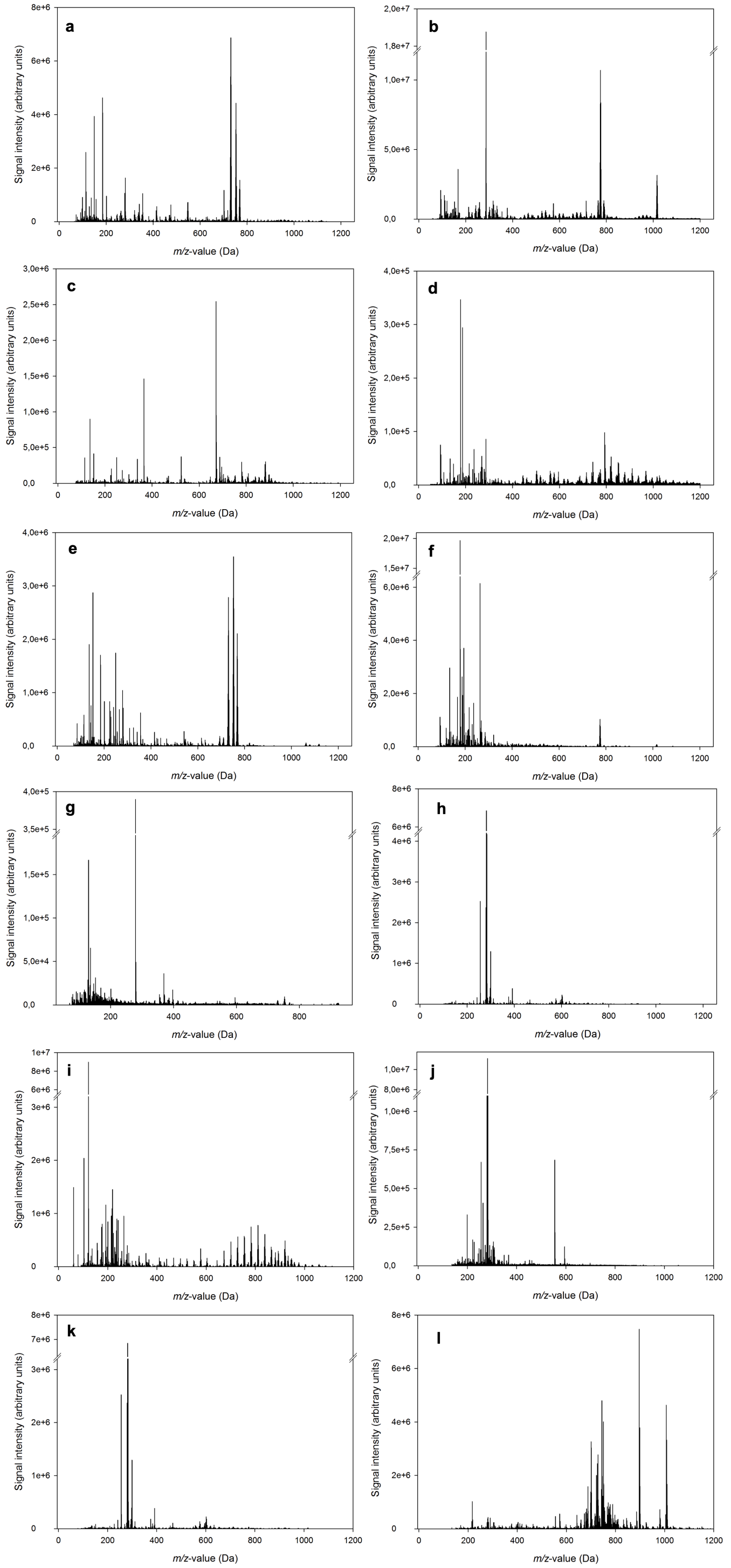


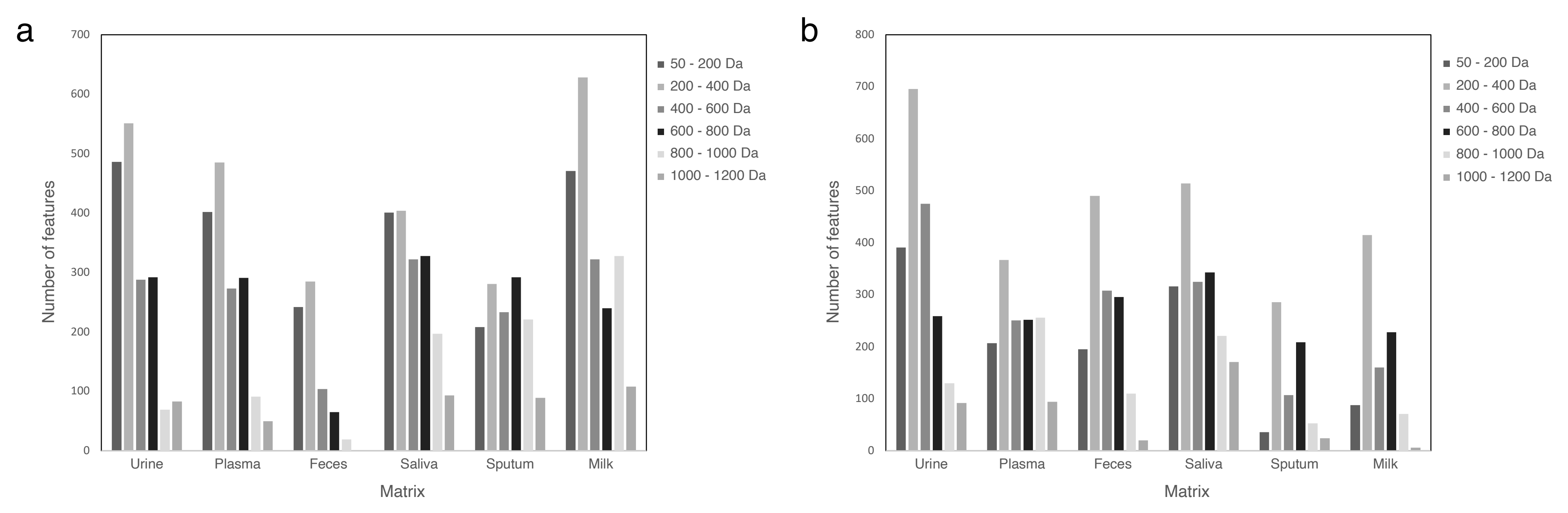


